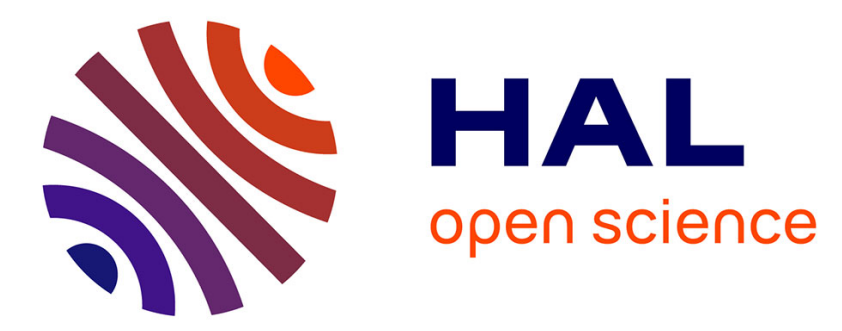

\title{
Minimum-Fuel Fixed-Time Impulsive Elliptic Glideslope Guidance Algorithms Using Semidefinite Programming
}

\author{
Yassine Ariba, Denis Arzelier, Laura Sofia Urbina
}

\section{To cite this version:}

Yassine Ariba, Denis Arzelier, Laura Sofia Urbina. Minimum-Fuel Fixed-Time Impulsive Elliptic Glideslope Guidance Algorithms Using Semidefinite Programming. Journal of Guidance, Control, and Dynamics, 2018, 41 (9), pp.1873 - 1887. 10.2514/1.G003395 . hal-01887840

\author{
HAL Id: hal-01887840 \\ https://hal.laas.fr/hal-01887840
}

Submitted on 4 Oct 2018

HAL is a multi-disciplinary open access archive for the deposit and dissemination of scientific research documents, whether they are published or not. The documents may come from teaching and research institutions in France or abroad, or from public or private research centers.
L'archive ouverte pluridisciplinaire HAL, est destinée au dépôt et à la diffusion de documents scientifiques de niveau recherche, publiés ou non, émanant des établissements d'enseignement et de recherche français ou étrangers, des laboratoires publics ou privés. 


\title{
Minimum-Fuel Fixed-Time Impulsive Elliptic Glideslope Guidance Algorithms Using Semidefinite Programming
}

\author{
Yassine Ariba* \\ LAAS-CNRS, Université de Toulouse; ICAM, 31031 Toulouse, France \\ Denis Arzelier ${ }^{\dagger}$, Laura Sofia Urbina-Iglesias \\ LAAS-CNRS, Université de Toulouse, CNRS, 31031 Toulouse, France
}

\begin{abstract}
A new minimum-fuel glideslope guidance algorithm for approaching autonomously a target evolving on an elliptic orbit is proposed in this paper. Assuming chemical propulsion, the present work aims at enhancing Hablani's seminal method. Although this reference method is efficient and easy to implement, there is no direct control on the fuel consumption and the guidance error. By identifying some relevant degrees of freedom, a new formulation of the glideslope guidance algorithm is proposed to address these issues. For a fixed-time rendezvous and a given number of maneuvers, the fuel-optimal multipulse glideslope is expressed as a semidefinite programming problem. A solution to this optimization problem provides an impulsive control sequence that guarantees a minimal consumption and makes sure the chaser's trajectory remains included inside a user-defined corridor. Besides, if trajectory constraints are removed, or if a specific direction is considered (V-bar or R-bar), then the formulation is reduced to a simple linear programming problem. Three realistic scenarios of rendezvous are simulated to illustrate the benefit of the proposed methodology.
\end{abstract}

\section{Nomenclature}

- $a=$ semi-major axis of the reference orbit;

- $e=$ eccentricity of the reference orbit;

$-v=$ true anomaly of the reference orbit;

- $\mu=$ the standard gravitational parameter;

- $n=\sqrt{\frac{\mu}{a^{3}}}=$ the mean motion of the reference orbit;

- $\Phi\left(t_{k+1}, t_{k}\right)=\phi\left(t_{k+1}\right) \phi^{-1}\left(t_{k}\right)=\Phi^{[k]}=$ transition matrix of relative motion;

- $N=$ number of maneuvers;

- $\Delta \vec{V}_{i}=$ velocity increment vector at $t_{i}$;

\footnotetext{
*Associate Professor, LAAS-CNRS, ICAM, yassine.ariba@icam.fr.

${ }^{\dagger}$ Researcher CNRS, LAAS-CNRS, arzelier@laas.fr.

${ }^{\ddagger}$ Ph.D. student CNRS, LAAS-CNRS, 1surbina@laas.fr.
} 
- $\Delta v_{i}=$ velocity impulse modulus at $t_{i}$;

- $\mathbb{R}\left[x_{1}, \cdots, x_{n}\right]$ stands for the algebra of polynomials in variables $\left(x_{1}, \cdots, x_{n}\right)$ with coefficients in $\mathbb{R}$;

- $\mathbb{O}_{p \times m}=$ the null matrix of dimensions $p \times m$;

- $\mathcal{I}_{m}=$ the identity matrix of dimension $m$;

- $\mathbb{1}_{m}=$ the $m$-vector composed of ones;

\section{Introduction}

Rendezvous (RDV) between two spacecraft (a target and a chaser) has been one of the most salient operational technology since its first manual achievement in the sixties between a Gemini vehicle and an unmanned target vehicle. Recently, an increasing demand is witnessed to perform autonomous rendezvous so as to address various proximity operations between an active spacecraft and a passive one : on-orbit servicing, refueling, repairing, de-orbiting. The references [1], [2], [3] among many others give specific examples of missions involving the rendezvous as a key technology.

The reference [4] defines the rendezvous process as a sequence of orbital maneuvers and guidance to drive the active spacecraft (chaser) into the vicinity of the passive spacecraft (target). This present work focuses on the close range rendezvous phase whose objective is to reduce the relative distance to the target before the entry into the final approach corridor. During this phase, relative navigation is used and the general objective of the control system is to ease a safe approach to the target spacecraft for the next proximity operations. If the chaser is assumed to be actuated by six independent ungimbaled chemical thrusters, then the control system is able to produce an instantaneous incremental change of the spacecraft's velocity vector in any direction while the position remains constant. This feature has led to several results based on impulsive maneuvers and various strategies have been proposed to execute the closing flight as a series of hops in a corridor [4] [5], [6], either via the tangential direction, called $V$-bar, or via the radial direction, called $R$-bar. Hops refer to the chaser's free trajectory between two successive impulsive maneuvers. Basically, each thrust is obtained by computing the velocity vector required to join the next specified location after some known duration of free motion. These calculations are a direct application of the transition matrix associated to the Clohessy-Wiltshire (CW) equations. Then, the incremental velocity impulse to be applied equals the difference between the computed velocity and the current velocity resulting from the previous hop.

A more general scheme is proposed by the glideslope approach that guides the spacecraft toward any direction [7], [8]. The glideslope approach is a straight line approach, defined in the Local Vertical Horizontal (LVLH) frame, from the initial position of the chaser to a final position close to the target spacecraft. The suitable velocity increments are computed according to range and range rate profiles defined by the user with almost the same computational process as in the classical V-bar or R-bar approaches. Note that this generalization of the previous approaches is particularly interesting in the case where the target has to keep a torque equilibrium attitude [9]. The off-axis glideslope 
approach has been first defined in the past for rendezvous and proximity operations involving the space shuttle [7]. This preliminary study, restricted to an in-plane motion with canted thrusters, has been extended for any direction in space in [8] (defining the so-called classical glideslope algorithm), reviewed in [10] and finally extended to elliptical orbits in [11] using the closed-form solution of the Tschauner-Hempel (TH) equations. However, a direct control on the fuel consumption imposed by a restricted propellant budget and on the guidance error inherent to hopping trajectories has been explicitly considered in none of these references.

In this paper, the classical glideslope algorithm [8] is revisited and a new approach is proposed to include optimization features in the design. Indeed, the usual methodology generates an impulsive maneuver sequences according to a predefined velocity profile that sets all positions where impulsive maneuvers will occur. As a result, the trajectory is entirely determined and the consumption and the excursion are by-products of the whole procedure. For that reason, we propose a new formulation of the glideslope guidance which aims at identifying relevant degrees of freedom. Getting some degrees of latitude allows to derive an optimization algorithm to minimize the fuel consumption. Besides, in all impulsive approaches mentioned above, the chaser's trajectory exhibits hops between maneuvers that are inherent to the natural relative motion of the spacecraft. Inspired from the result of [12], translating a constraint on the trajectory to a linear matrix inequality (using a suitable parametrization of the Yamanaka-Ankersen equations [13], a change of variable and a mathematical result on non-negative polynomials [14]), a new methodology is proposed to control the amplitude of hops. This feature is a desirable asset since it enforces the chaser to remain close enough to the commanded path. It becomes even essential if a line-of-sight (LoS) constraint is required to keep the spacecraft into the visibility cone of the target's sensors. In addition, the optimization problem formulation also enables us to readily take the maximum thrust of the chaser actuators into account. It is worth noting that while the classical glidelope guidance of [8] is limited to circular orbits, the proposed version extends naturally to elliptical orbits. Moreover, the transfer time in the former approach is indirectly derived from the choice of the velocity profile, whereas it is a user-defined input in the present one and leads to a genuine fixed-time rendezvous approach. The contribution of this work is summarized as follows.

1) Contrary to the classical glidelope approach, the proposed guidance algorithm is designed with the TH equations, and thus is also workable for eccentric orbits.

2) The new glidelope algorithm results in a semidefinite optimization problem that minimizes the fuel consumption.

3) The trajectory hops of the chaser are enclosed into a user-defined corridor.

The resulting algorithm for off-axis glideslope is expressed as a semidefinite programming problem (SDP), which can be solved efficiently. This formulation can be further reduced to end into a linear programming problem (LP) when no constraints on the trajectory are considered or when the guidance is limited to a V-bar or R-bar approach. Three numerical examples illustrate the benefit of the proposed methodology and compare it with other approaches from the literature. Two criteria are considered to assess performances of algorithms : the consumption and the guidance error 
w.r.t. the glideslope reference.

The present article encompasses the results and material of the conference papers [15] and [16] with additional technical developments and different numerical examples.

\section{The Classical glideslope approach algorithm}

\section{A. Relative motion dynamics}

The close range phase of the spacecraft rendezvous mission is characterized by the use of relative navigation since the separation between spacecraft is sufficiently small [4]. In that case, the relative motion of the chaser is expressed in the Local-Vertical-Local-Horizontal (LVLH) frame. The origin of the coordinate frame is located at the center of mass of the target and the space is spanned by $\left(\vec{X}_{o t}, \vec{Y}_{o t}, \vec{Z}_{o t}\right)$ where the $\vec{Z}_{o t}$ axis is in the radial direction (R-bar) oriented towards the center of the Earth, the $\vec{Y}_{o t}$ axis is perpendicular to the leader orbital plane and pointing in the opposite direction of the angular momentum (H-bar) while the $\vec{X}_{o t}$ axis completes the right-hand triad $\vec{X}_{o t}=\vec{Y}_{o t} \times \vec{Z}_{o t}$ (V-bar, see Figure 11. In Figure 1, the vector $\vec{\rho}(t)$ defines the relative position of the chaser with respect to the target at time $t$. Under Keplerian assumptions (no orbital perturbations are considered) and an elliptic reference orbit, the equations

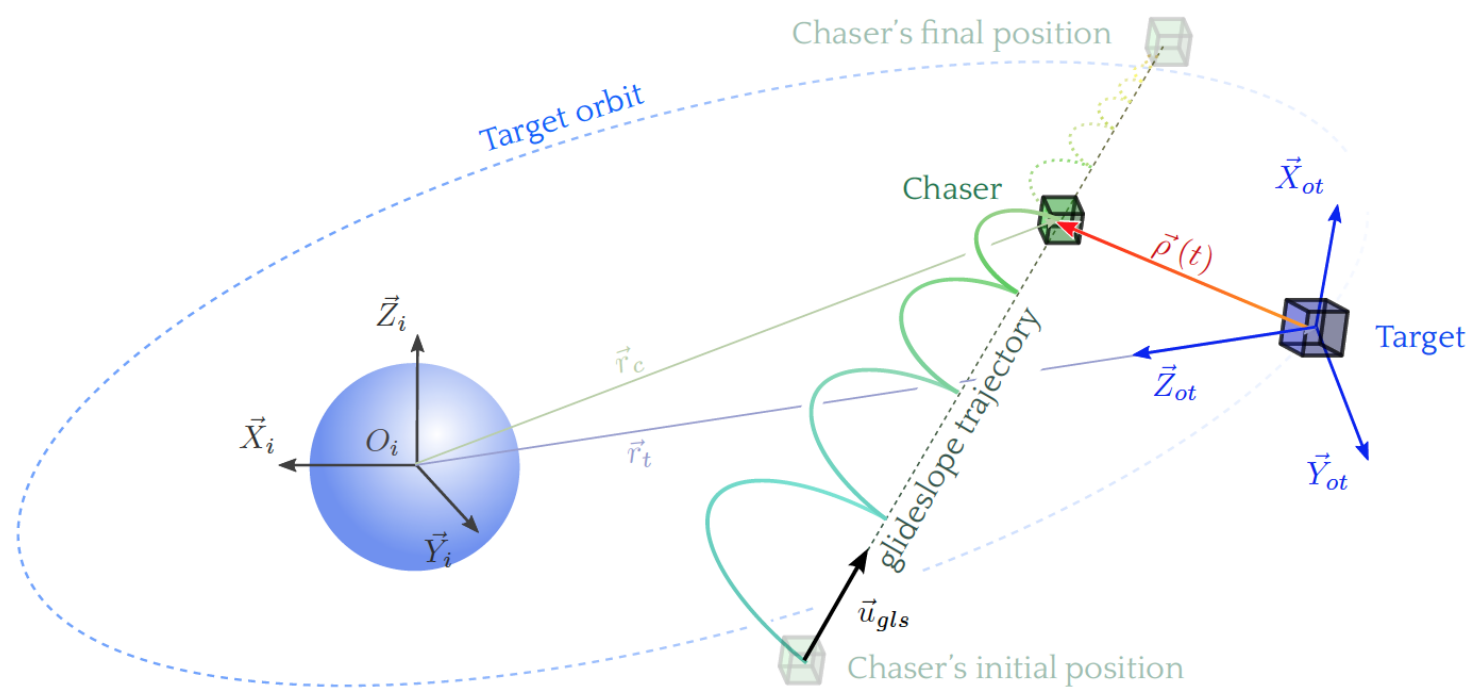

Figure 1 Glideslope approach (not to scale).

of motion for the relative motion in the LVLH frame may be linearized for close separation between the target and the chaser [17, Chapter 5, Section 5.6.1], leading to the state-space representation:

$$
\dot{\vec{X}}(t)=A_{0}(t) \vec{X}(t)+\left[\begin{array}{c}
0_{3 \times 3} \\
I_{3},
\end{array}\right] \frac{\vec{f}(t)}{m_{F}}
$$


where state $\vec{X}(t)=\left[\vec{\rho}^{T}(t) \vec{v}^{T}(t)\right]^{T}$ represents the relative position and velocity of the chaser in the LVLH frame, $f(t)=\left[f_{x}(t) f_{y}(t) f_{z}(t)\right]^{T}$ is the thrust vector, $m_{F}$ is the mass of the chaser and the dynamic matrix $A_{0}(t)$ is a periodic matrix of time $t$ given by :

$$
A_{0}(t)=\left[\begin{array}{cccccc}
0 & 0 & 0 & 1 & 0 & 0 \\
0 & 0 & 0 & 0 & 1 & 0 \\
0 & 0 & 0 & 0 & 0 & 1 \\
a_{1}(t) & 0 & \ddot{v} & 0 & 0 & 2 \dot{v} \\
0 & a_{2}(t) & 0 & 0 & 0 & 0 \\
-\ddot{v} & 0 & a_{3}(t) & -2 \dot{v} & 0 & 0
\end{array}\right] .
$$

Here,

$$
a_{1}(t)=\dot{v}^{2}-k^{4} \kappa^{3}(v), \quad a_{2}(t)=-k^{4} \kappa^{3}(v), \quad a_{3}(t)=\dot{v}^{2}+2 k^{4} \kappa^{3}(v),
$$

by noting that:

$$
\frac{d v}{d t}=\frac{n}{\left(1-e^{2}\right)^{3 / 2}}(\underbrace{1+e \cos v}_{\kappa(v)})^{2}=: k^{2} \kappa^{2}(v),
$$

where $n=\sqrt{\frac{\mu}{a^{3}}}=2 \pi / T$ is the mean motion of the leader orbit, satisfying for any fixed $v_{0}, t_{0}$ :

$$
v-v_{0}=2 \pi \quad \Rightarrow \quad n\left(t-t_{0}\right)=2 \pi
$$

It is assumed that only the chaser is active and actuated using 6 ungimbaled identical chemical thrusters. The use of chemical propulsion leads to idealize possible thrusts as impulsive maneuvers providing instantaneous velocity jumps in the three axes while the relative position remains unchanged during firing. The impulsive control input is thus defined as:

$$
\Delta \vec{V}\left(t_{k}\right):=\Delta \vec{V}_{k}:=\int_{t_{k}^{-}}^{t_{k}^{+}} \frac{1}{m_{F}}\left[\begin{array}{c}
f_{x}(t) \\
f_{y}(t) \\
f_{z}(t)
\end{array}\right] \mathrm{d} t
$$

where $t_{k}$ is a generic firing time and $\Delta \vec{V}_{k}$ represents the applied impulsive thrust. In order to compute the transition matrix $\Phi\left(t, t_{0}\right)$ for the linearized Equations (1) and (2), classical derivations dating back to the seminal publications of Lawden [18, Chapter 5] and Tschauner-Hempel [19] consists in applying a change of independent variable from time 
$t$ to true anomaly $v$ and a simplifying coordinate change leading to $\overrightarrow{\tilde{X}}(v)=T(v) \vec{X}(t)$ with:

$$
T(v):=\left[\begin{array}{cc}
\kappa(v) \mathcal{I}_{3} & \mathbb{D}_{3 \times 3} \\
\kappa(v)^{\prime} \mathcal{I}_{3} & \frac{1}{k^{2} \kappa(v)} \mathcal{I}_{3}
\end{array}\right] .
$$

A simplified autonomous state space representation $\overrightarrow{\tilde{X}}^{\prime}(v)=\tilde{A}(v) \overrightarrow{\tilde{X}}(v)$ is obtained with:

$$
\tilde{A}(v)=\left[\begin{array}{cccccc}
0 & 0 & 0 & 1 & 0 & 0 \\
0 & 0 & 0 & 0 & 1 & 0 \\
0 & 0 & 0 & 0 & 0 & 1 \\
0 & 0 & 0 & 0 & 0 & 2 \\
0 & -1 & 0 & 0 & 0 & 0 \\
0 & 0 & \frac{3}{\kappa(v)} & -2 & 0 & 0
\end{array}\right] .
$$

Based on a particular fundamental solution $\tilde{\varphi}_{v_{0}}(v)$, the so-called Yamanaka-Ankersen form of the transition matrix $\tilde{\Phi}\left(v, v_{0}\right)$ has been proposed in the reference [13]. This form is particularly interesting regarding the computing efficiency and the transition matrix $\Phi\left(t, t_{0}\right)$ may be easily computed by:

$$
\Phi\left(t, t_{0}\right)=T(v)^{-1} \tilde{\varphi}_{\nu_{0}}(v) \tilde{\varphi}_{\nu_{0}}\left(v_{0}\right) T\left(v_{0}\right)
$$

Thus, a controlled trajectory composed of $N+1$ impulses is described by the following equation:

$$
\vec{X}(t)=\Phi\left(t, t_{0}\right) \vec{X}\left(t_{0}\right)+\sum_{k=0}^{N} \Phi\left(t, t_{k}\right) B \Delta \vec{V}_{k}
$$

where $t_{1}<t_{2}<\cdots<t_{N} \leq t$ and $\Delta \vec{V}_{k}$ is the impulsive control applied at $t_{k} . \quad B=\left[\mathbb{O}_{3 \times 3} I_{3}\right]^{T}$ is the input matrix. Hereafter, the following notation describing the free motion with a block partitioned transition matrix is adopted:

$$
\vec{X}(t)=\left[\begin{array}{c}
\vec{\rho}(t) \\
\hline \vec{v}(t)
\end{array}\right]=\left[\begin{array}{l|l}
\Phi_{\rho \rho}\left(t, t_{0}\right) & \Phi_{\rho v}\left(t, t_{0}\right) \\
\hline \Phi_{v \rho}\left(t, t_{0}\right) & \Phi_{v v}\left(t, t_{0}\right)
\end{array}\right]\left[\begin{array}{c}
\vec{\rho}_{0} \\
\hline \vec{v}_{0}
\end{array}\right] .
$$

The analytical expressions of each partition of the state transition matrix $\Phi\left(t, t_{0}\right)$ in the context of an elliptical orbit are not given here, since they have a convoluted analytical form and all we need is to know how to compute them efficiently.

However, in the case of a circular orbit, the relative dynamics are governed by the Hill-Clohessy-Wiltshire equations 
[20], leading to a simpler expression of the four partitions of $\Phi\left(t, t_{0}\right) \in \mathbb{R}^{6 \times 6}$.

\section{B. Hablani's classical glideslope approach for rendezvous}

When considering design of impulsive maneuvers for a glideslope rendezvous, the most cited reference is the paper by [8], in which the so-called classical inbound and outbound glideslope approaches for a circular reference orbit are presented in a general setup. When the chaser must approach the target satellite, an inbound glideslope guidance algorithm is used. On the other hand, an outbound algorithm is used for receding away from the target. In both cases, the chaser's relative velocity must be low when it is close to the target, in order to comply with safety constraints. The real trajectory of the chaser will not actually be a straight line, but will be composed of a sequence of humps between the different thrust locations on the glideslope line, as shown in Figure 1 These humps appear because of the effect of the gravitational forces acting over the chaser and of the applied impulses, and will vary according to the type of orbit. When performing a glideslope approach, the chaser will depart from an initial position $\vec{\rho}\left(t_{0}\right)=\vec{\rho}_{0}=\left[\begin{array}{lll}x_{0} & y_{0} & z_{0}\end{array}\right]^{T}$ and will arrive at a final position $\vec{\rho}\left(t_{f}\right)=\vec{\rho}_{f}=\left[\begin{array}{lll}x_{f} & y_{f} & z_{f}\end{array}\right]^{T}$ at a fixed final time $t_{f}$. This final position can be a station keeping position, a point that belongs to a given periodic reference trajectory or a docking point. In order to parameterize the rectilinear trajectory to be followed by the chaser — also known as commanded path $[8]-$ from $\vec{\rho}_{0}$ to $\vec{\rho}_{f}$, the vector $\vec{\lambda}(t)$ is introduced, whose origin is located at the chaser's final position and pointing the chaser's current position, as in Figure 2, thus verifying:

$$
\vec{\lambda}(t)=\vec{\rho}_{g l s}(t)-\vec{\rho}_{f}
$$

where $\vec{\rho}_{g l s}(t)$ is the relative position vector of the orthogonal projection of the chaser on the glideslope line with respect to the target. The unit vector of the rectilinear trajectory's direction is expressed as:
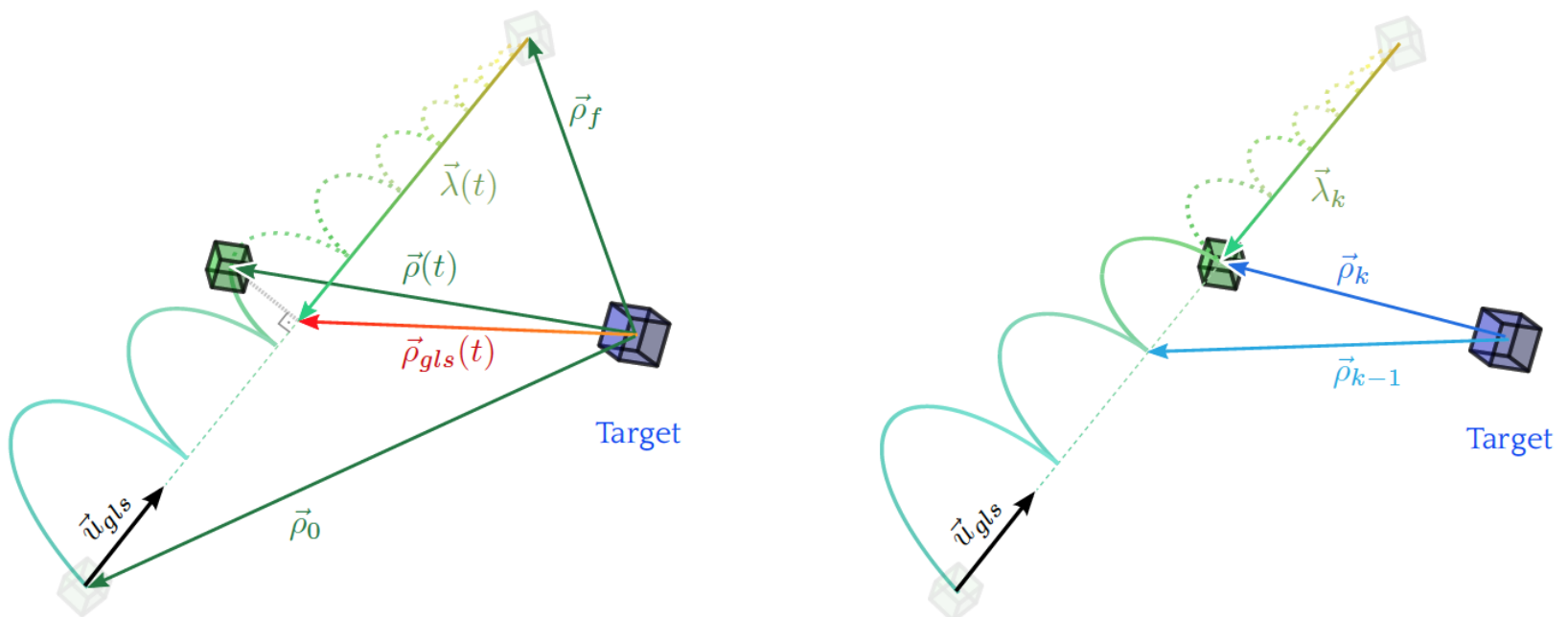

Figure 2 General position. 


$$
\vec{u}_{g l s}=\left[\begin{array}{lll}
\frac{x_{f}-x_{0}}{\left\|\vec{\rho}_{f}-\vec{\rho}_{0}\right\|} & \frac{y_{f}-y_{0}}{\left\|\vec{\rho}_{f}-\vec{\rho}_{0}\right\|} & \frac{z_{f}-z_{0}}{\left\|\vec{\rho}_{f}-\vec{\rho}_{0}\right\|}
\end{array}\right] .
$$

Note that the commanded path vector $\vec{\lambda}_{f}$ at final time $t_{f}$ is zero by definition:

$$
\vec{\lambda}_{f}=\overrightarrow{0}
$$

At every instant $t$, the distance to go is a function of time, and is given by $\lambda(t)=\|\vec{\lambda}(t)\|$ in the direction of the unit vector $\vec{u}_{g l s}$ :

$$
\vec{\lambda}(t)=-\lambda(t) \vec{u}_{g l s}
$$

The main features of the classical glideslope algorithm from [8] are now recalled. The chaser is commanded to reach $\vec{\rho}_{f}$ from $\vec{\rho}_{0}$ following a specific linear commanded velocity profile:

$$
\dot{\lambda}=\alpha \lambda+\dot{\lambda}_{f}
$$

where $\alpha$ is the slope of $\dot{\lambda}$ vs $\lambda$. The initial distance to go $\lambda_{0}$, the initial commanded profile velocity $\dot{\lambda}_{0}<0$ and the final arrival profile velocity $\dot{\lambda}_{f}<0$ are quantities specified by the designer, required to define the slope $\alpha$ :

$$
\alpha=\frac{\dot{\lambda}_{0}-\dot{\lambda}_{f}}{\lambda_{0}}<0
$$

The profile for the distance to go $\lambda(t)$ is therefore readily deduced as:

$$
\lambda(t)=\lambda_{0} e^{\alpha t}+\frac{\dot{\lambda}_{f}}{\alpha}\left(e^{\alpha t}-1\right) .
$$

Note that $\dot{\lambda}_{0}>\dot{\lambda}_{f}$ since a decelerating glideslope trajectory is sought. For a given set of these parameters, the basic principle of the classical algorithm is then to analytically compute a fixed number of impulses equally spaced in time over the transfer duration $T$, leading to a constant interval between two consecutive thrusts:

$$
\Delta t=\frac{T}{N} .
$$

The thrusters will be then fired at times:

$$
t_{k}=k \Delta t, \quad k=0,1, \cdots, N-1 .
$$


Each incremental velocity control at $\vec{\rho}_{k}$ is computed as $\Delta \vec{V}_{k}=\vec{v}_{k_{+}}-\vec{v}_{k_{-}}$where $\vec{v}_{k_{+}}$is the departure velocity needed to go from $\vec{\rho}_{k}$ to $\vec{\rho}_{k+1}$ and $\vec{v}_{k_{-}}$is the arrival velocity at $\vec{\rho}_{k}$. In order to obtain the maneuver plan composed by the $N$ impulses $\left\{\Delta \vec{V}_{0}, \Delta \vec{V}_{1}, \cdots, \Delta \vec{V}_{k}, \cdots, \Delta \vec{V}_{N-1}\right\}$, we need the relative velocities right before and just after the thrust. Both quantities are simply obtained by solving the autonomous equations (10) at each step $k$, since:

$$
\begin{aligned}
& \vec{\rho}_{k+1}=\Phi_{\rho \rho}\left(t_{k+1}, t_{k}\right) \vec{\rho}_{k}+\Phi_{\rho v}\left(t_{k+1}, t_{k}\right) \vec{v}_{k_{+}}, \\
& \vec{v}_{k+1_{-}}=\Phi_{v \rho}\left(t_{k+1}, t_{k}\right) \vec{\rho}_{k}+\Phi_{v v}\left(t_{k+1}, t_{k}\right) \vec{v}_{k+},
\end{aligned}
$$

and:

$$
\vec{v}_{k_{+}}=\Phi_{\rho v}\left(t_{k+1}, t_{k}\right)^{-1}\left[\vec{\rho}_{k+1}-\Phi_{\rho \rho}\left(t_{k+1}, t_{k}\right) \vec{\rho}_{k}\right]
$$

which allows the chaser to arrive at the next position $\vec{\rho}_{k+1}$ with relative velocity $\vec{v}_{k+1}$ at time $t_{k+1}$. Recall that in the classical approach, transition matrices are actually based on the HCW equations, restricted to circular orbits, and depend only on the difference $t_{k+1}-t_{k}$.

The classical glideslope algorithm is straightforward and easy to implement but suffers from key shortcomings. First, it is limited to circular reference orbits. Second, it is important to remind that the actual trajectory of the chaser will not be strictly along the commanded straight line path but will exhibit humps between the $N$ points where an impulsive maneuver is performed and located on the commanded path (cf. Figure 2). In addition, if the initial and final commanded velocities of the glideslope profile are a priori given, there is no degree of freedom left to control the transfer time and the consumption. Indeed, the transfer time $T$ is not fixed a priori but deduced from the initial and final commanded velocities $\left(\dot{\lambda}_{0}, \dot{\lambda}_{f}\right)$ and from the initial distance to go $\lambda_{0}$ :

$$
T=\frac{1}{\alpha} \ln \left[\frac{\dot{\lambda}_{f}}{\dot{\lambda}_{0}}\right]=\frac{\lambda_{0}}{\dot{\lambda}_{0}-\dot{\lambda}_{f}} \ln \left[\frac{\dot{\lambda}_{f}}{\dot{\lambda}_{0}}\right]
$$

The consumption itself is computed a posteriori from the velocity increments without any possibility to optimize it for given side conditions of the rendezvous. The objective of the present paper is therefore to propose a new optimization algorithm for the general glideslope framework and extend the results to elliptic reference orbits, taking these two important features into account:

- Minimize the fuel-consumption for a given set of initial and final rendezvous conditions and an a priori fixed time of transfer;

- Control the maximum guidance error by defining constraints on the humps profile. 


\section{Minimum-fuel glideslope approach with controlled humps}

The main result of the paper is now presented. It mainly consists in identifying some degrees of freedom and deriving a numerically tractable expression for the different constraints on the chaser trajectory and to minimize the fuel consumption during the glideslope transfer. First, the glideslope line tracking constraints are defined according to the transition matrix of the relative linearized keplerian elliptic dynamics. Contrasting with to [8] or [11], intermediate positions are free variables and a constraint is added to control the final relative velocity of the chaser. Next, the constraints on the humps profile are dealt with using a parametrization of the relative trajectory defined in [12] and results from polynomial optimization from the reference [14]. Finally, a general minimum-fuel multipulse glideslope guidance algorithm relying on the solution of a semidefinite programming problem is proposed.

\section{A. Glideslope line tracking}

In order to perform the transfer from $\vec{\rho}_{0}$ to $\vec{\rho}_{f}$ in a given duration $T$, the number of thruster firings is fixed and equal to $N+1$. Any two successive impulsive maneuvers are separated by $\Delta t=T / N$ and impulsive thrusts are applied at dates $t_{k}=k \Delta t, k=0,1, \cdots, N$. Note that our approach does not actually require maneuvers to be equally spaced in time. Only the time grid, $t_{k}$ for $k=0,1, \cdots, N$, needs to be specified. Throughout the transfer, the spacecraft must follow the commanded path. After each maneuver, the chaser must be back on the glideslope line. The initial $\vec{\rho}_{0}$ and final $\vec{\rho}_{N}=\vec{\rho}_{f}$ positions are fixed by specifications. Intermediate positions are set free and are parameterized as:

$$
\vec{\rho}_{k}=\vec{\rho}_{0}+\lambda_{k} \vec{u}_{g l s}, k=0, \ldots, N
$$

The scalars $\lambda_{k}$ are free and denote here the traveled distance from $\vec{\rho}_{0}$ to $\vec{\rho}_{k}$. Note that $\lambda_{0}=0$ and $\lambda_{N}=\lambda_{f}=\left\|\vec{\rho}_{f}-\vec{\rho}_{0}\right\|$. A set of $N$ equations of the form:

$$
\vec{\rho}_{k+1}=\Phi_{\rho \rho}^{[k]} \vec{\rho}_{k}+\Phi_{\rho v}^{[k]} \vec{v}_{k_{+}}, k=0, \ldots, N-1,
$$

with the position vectors $\vec{\rho}_{k+1} / \vec{\rho}_{k}$, describes the transfer of the chaser after the impulsive actuation at $t_{k}$. $\vec{v}_{k_{+}}$is the velocity vector right after the impulse is applied. Combining Equations (24) and (25) enforces the requirement for the chaser to come back on the path after each maneuver period, leading to the set of equations:

$$
\begin{aligned}
& \lambda_{k+1} \vec{u}_{g l s}-\lambda_{k} \Phi_{\rho \rho}^{[k]} \vec{u}_{g l s}-\Phi_{\rho \nu}^{[k]} \vec{v}_{k_{+}}=\left(\Phi_{\rho \rho}^{[k]}-\mathcal{I}_{3}\right) \vec{\rho}_{0}, \\
& \lambda_{1} \vec{u}_{g l s}-\Phi_{\rho v}^{[0] \vec{v}_{0_{+}}}=\left(\Phi_{\rho \rho}^{[0]}-\mathcal{I}_{3}\right) \vec{\rho}_{0},
\end{aligned}
$$

for $k=1, \cdots, N-1$. Because the reference orbit is considered to be elliptic, the transition matrix is not constant all over the orbit and needs to be updated for each maneuver. Since time intervals for impulse control are input data, all 
transition matrices $\Phi^{[k]}$ can be computed a priori. The decision variables in 26 are composed by the sequence of scalar variables $\lambda_{k}$ for $k=1, \cdots, N-1$ and by the sequence of vectors $\vec{v}_{k_{+}}$for $k=0,1, \cdots, N-1$. The sequence of impulses is deduced afterwards, computing the difference between the design variable $\vec{v}_{k_{+}}$and the velocity vector $\vec{v}_{k_{-}}$ resulting from the previous maneuver and from the relative dynamics of the chaser:

$$
\vec{v}_{k+1_{-}}=\Phi_{v \rho}^{[k]} \vec{\rho}_{k}+\Phi_{v v}^{[k]} \vec{v}_{k_{+}}, k=0,1, \cdots, N-1
$$

Therefore, we have

$$
\Delta \vec{V}_{k}=\vec{v}_{k_{+}}-\vec{v}_{k_{-}} .
$$

\section{B. Final velocity constraint}

Since a last impulse is needed to control the final velocity of the spacecraft, an additional equality constraint is defined. This $(N+1)^{t h}$ impulse maneuver is given by:

$$
\begin{aligned}
\Delta \vec{V}_{N} & =\vec{v}_{N_{+}}-\vec{v}_{N_{-}} \\
\Delta \vec{V}_{N} & =\vec{v}_{N_{+}}-\Phi_{v \rho}^{[N-1]} \vec{\rho}_{N-1}-\Phi_{v v}^{[N-1]} \vec{v}_{N-1_{+}} .
\end{aligned}
$$

Setting the vector $\vec{v}_{N_{+}}=\vec{v}_{f}$ as the desired final velocity and $\Delta \vec{V}_{N}$ being a free variable, an extra equality constraint is appended:

$$
\vec{v}_{f}-\Phi_{v \rho}^{[N-1]} \vec{\rho}_{0}=\Phi_{v \rho}^{[N-1]} \vec{u}_{g l s} \lambda_{N-1}+\Phi_{v v}^{[N-1]} \vec{v}_{N-1_{+}}+\Delta \vec{V}_{N}
$$

As mentioned earlier, $\Delta \vec{V}_{N}, \lambda_{N-1}$ and $\vec{v}_{N-1_{+}}$are the only free variables of 30 .

\section{Constraints on guidance error}

The aim of this subsection is to give a numerically tractable formulation of the continuous constraints imposed on the spacecraft relative trajectory in order to bound the guidance error inherent to the impulsive glideslope approach. In the spirit of the method developed in [12], the idea is to look for an equivalent finite description of the admissible relative trajectories using various tools from algebraic geometry and in particular, properties of non negative polynomials. The main steps of the method are: 1) define a piecewise linear envelope enclosing the admissible trajectory; 2) use a rational parametrization of the trajectory between each pulse to transform the previous continuous linear constraints into polynomials non negativity constraints; 3) apply representation theorems of cones of nonnegative polynomials from [14] to get a final semidefinite formulation of the constraints on guidance error. 


\section{Admissible trajectories envelope}

First, a set of linear constraints on the chaser's relative trajectory is defined for each maneuver interval $\left[t_{k} t_{k+1}\right]$, defined as the elapsed time between the $k^{\text {th }}$ impulse to the instant when the spacecraft is back on the glideslope line.

The input specifications regarding the maximal allowable excursion will define a rectangular corridor with four planes parallel to the glideslope direction $\vec{u}_{g l s}$ bounding the trajectory at each step $k$. We define the parameters $\delta_{\alpha_{k}}$ and $\delta_{\beta_{k}}$ to specify the distance from the glideslope line to each pair of planes, as shown in Figure $3 \mathrm{a}$

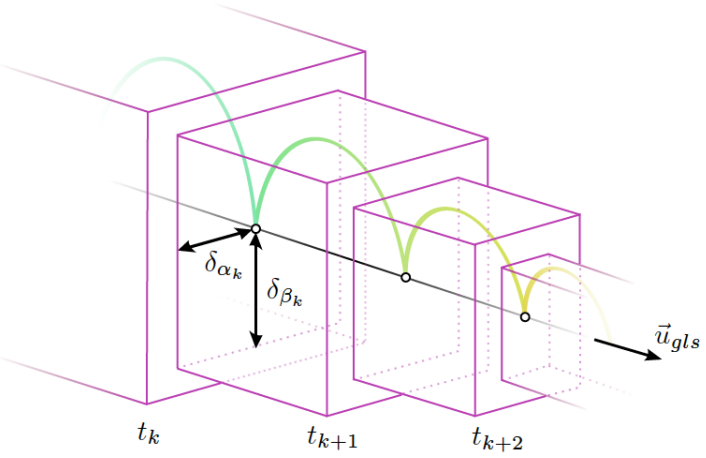

(a) Rectangular corridor bounding the trajectory.

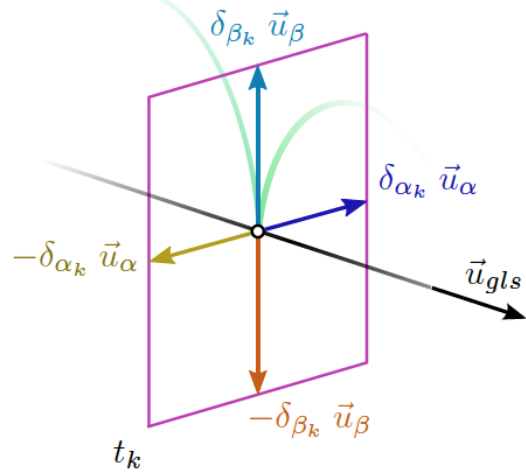

(b) Orthonormal basis to vector $\vec{u}_{g l s}$.

Figure 3 Glideslope corridor constraints.

A set of four linear constraints (upper and lower bounds for both parameters $\delta_{\alpha_{k}}$ and $\delta_{\beta_{k}}$ ) will be therefore necessary to define the maximum allowable excursion at each maneuver, represented by the following inequalities:

$$
\begin{aligned}
& -\vec{u}_{\alpha}^{T}\left(\vec{\rho}_{k}-\delta_{\alpha_{k}} \vec{u}_{\alpha}\right) \leq \vec{u}_{\alpha}^{T} \vec{\rho}(t) \leq \vec{u}_{\alpha}^{T}\left(\vec{\rho}_{k}+\delta_{\alpha_{k}} \vec{u}_{\alpha}\right), \\
& -\vec{u}_{\beta}^{T}\left(\vec{\rho}_{k}-\delta_{\beta_{k}} \vec{u}_{\beta}\right) \leq \vec{u}_{\beta}^{T} \vec{\rho}(t) \leq \vec{u}_{\beta}^{T}\left(\vec{\rho}_{k}+\delta_{\beta_{k}} \vec{u}_{\beta}\right),
\end{aligned}
$$

where $\vec{u}_{\alpha}$ and $\vec{u}_{\beta}$ are unit vectors, chosen as an orthonormal basis for the null space of $\vec{u}_{\text {gls }}^{T}$, as shown in Figure $3 \mathrm{~b}$ The inequalities in Expression (31) can be recasted in a matrix form as:

$$
A_{k} \vec{\rho}(t) \leq b_{k}, \forall t \in\left[t_{k} t_{k+1}\right], \forall k=0,1, \ldots, N-1,
$$

where $A_{k} \in \mathbb{R}^{n_{c} \times 3}$ is a constant matrix, $n_{c}$ denotes the number of scalar inequalities, each of which defines a plane 
bounding the trajectory ( $n_{c}=4$ in this case); and $b_{k} \in \mathbb{R}^{n_{c}}$ is a vector that depends on the user-defined parameters:

$$
\underbrace{\left[\begin{array}{c}
\vec{u}_{\alpha}^{T} \\
-\vec{u}_{\alpha}^{T} \\
\vec{u}_{\beta}^{T} \\
-\vec{u}_{\beta}^{T}
\end{array}\right]}_{A_{k}} \vec{\rho}(t) \leq \underbrace{\left[\begin{array}{cc}
\vec{u}_{\alpha}^{T}\left(\vec{\rho}_{k}+\delta_{\alpha_{k}}\right. & \left.\vec{u}_{\alpha}\right) \\
-\vec{u}_{\alpha}^{T}\left(\vec{\rho}_{k}-\delta_{\alpha_{k}}\right. & \left.\vec{u}_{\alpha}\right) \\
\vec{u}_{\beta}^{T}\left(\vec{\rho}_{k}+\delta_{\beta_{k}}\right. & \left.\vec{u}_{\beta}\right) \\
-\vec{u}_{\beta}^{T}\left(\vec{\rho}_{k}-\delta_{\beta_{k}}\right. & \left.\vec{u}_{\beta}\right)
\end{array}\right]}_{b_{k}},
$$

where the inequalitites are componentwise inequalities between vectors. Matrix $A_{k}$ is constant $\forall k=0,1, \ldots, N-1$, since all boxes are oriented along the glideslope line. However, vector $b_{k}$ might change according to the different distance specifications $\left\{\delta_{\alpha_{k}}, \delta_{\beta_{k}}\right\}$ associated to each maneuver $k$, as shown in Figure 3 a Applying the change of variable (6) to the general constraint 32 , we get:

$$
A_{k} \overrightarrow{\tilde{\rho}}(v) \leq \kappa(v) b_{k}, \quad \forall v \in\left[v_{k} v_{k+1}\right], \forall k=\{0, \ldots, N-1\}
$$

In [12], the autonomous relative trajectory $\overrightarrow{\tilde{\rho}}$ was parameterized as follows:

$$
\left\{\begin{array}{l}
\tilde{x}(v)=(2+e \cos v)\left(d_{1} \sin v-d_{2} \cos v\right)+d_{3}+3 d_{4} J\left(v, v_{k}\right)(1+e \cos v)^{2} \\
\tilde{y}(v)=d_{5} \cos v+d_{6} \sin v \\
\tilde{z}(v)=(1+e \cos v)\left(d_{2} \sin v+d_{1} \cos v\right)-3 e d_{4} J\left(v, v_{k}\right) \sin v(1+e \cos v)+2 d_{4}
\end{array}\right.
$$

for $v \in\left[v_{k}, v_{k+1}\right]$, where the vector of parameters $\vec{D}$ is defined by 37 and depends linearly on the initial state and the integral term $J\left(v, v_{k}\right)$ is given by:

$$
J\left(v, v_{k}\right)=\int_{v_{k}}^{v} \frac{1}{\kappa(u)^{2}} d u=\frac{n}{\left(1-e^{2}\right)^{3 / 2}}\left(t-t_{k}\right)
$$

\section{Rational parametrization of the trajectory constraints via polynomial non-negativity constraints}

Expression (35) shows several trigonometrical terms. The following change of variable is used in order to transform the trigonometrical functions into rational functions:

$$
w=\tan \frac{v}{2}, \cos v=\frac{1-w^{2}}{1+w^{2}}, \sin v=\frac{2 w}{1+w^{2}}
$$




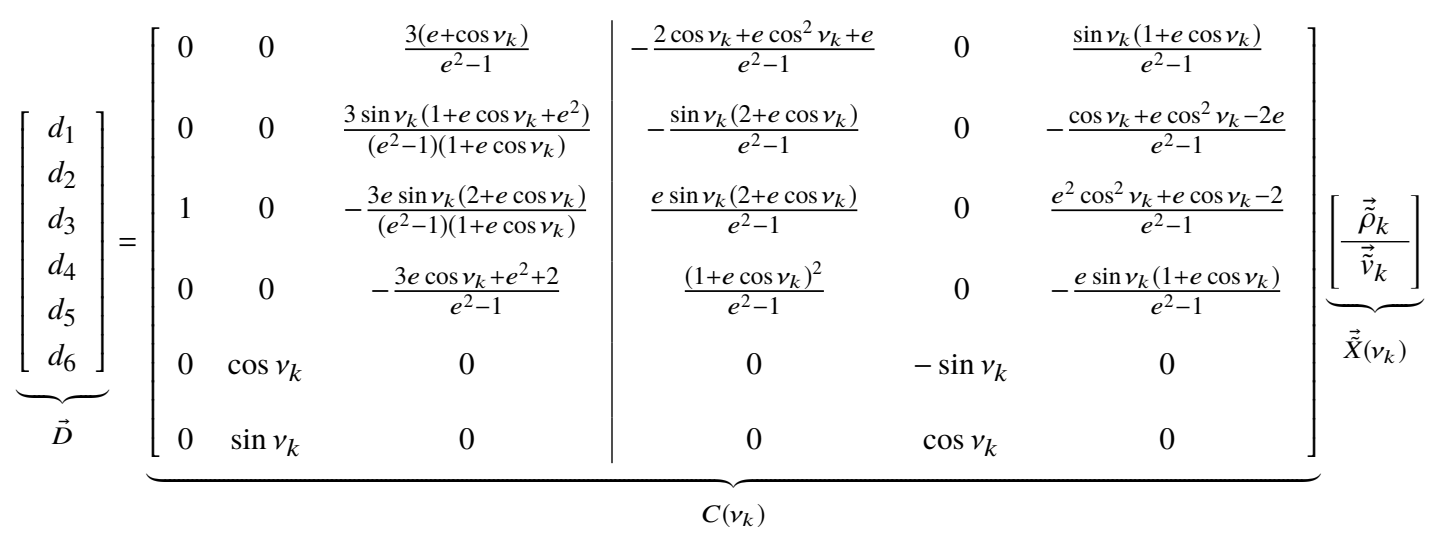

The propagation of the spacecraft relative motion can then be expressed as a function of $w$ :

$$
\left\{\begin{aligned}
\tilde{x}(w) & =\frac{1}{\left(1+w^{2}\right)^{2}}\left[P_{x}(w)+3 d_{4} P_{J x}(w) J(w)\right], \\
\tilde{y}(w) & =\frac{1}{\left(1+w^{2}\right)} P_{y}(w), \\
\tilde{z}(w) & =\frac{1}{\left(1+w^{2}\right)^{2}}\left[P_{z}(w)+2 d_{4} P_{J z}(w) J(w)\right]
\end{aligned}\right.
$$

for $w \in\left[w_{k}, w_{k+1}\right]$. Only the term with $J(w)$ is non-rational and requires to be dealt with. All $P_{*}$ functions are polynomials:

$$
\begin{aligned}
& P_{J x}(w)=\left((1+e)+(1-e) w^{2}\right)^{2}, \\
& P_{J z}(w)=-3 e\left((1+e) w+(1-e) w^{3}\right), \\
& P_{x}(w)=\sum_{i=0}^{4} p_{x i} w^{i}, P_{y}(w)=\sum_{i=0}^{2} p_{y i} w^{i}, P_{z}(w)=\sum_{i=0}^{4} p_{z i} w^{i},
\end{aligned}
$$

where coefficients of $P_{x}, P_{y}$ and $P_{z}$ depend linearly on the vector $\vec{D}$, and therefore depend linearly on the state $\overrightarrow{\tilde{X}}\left(v_{k}\right)$ at the firing instant $v_{k}$.

$$
\underbrace{\left[\begin{array}{c}
p_{x 0} \\
p_{x 1} \\
p_{x 2} \\
p_{x 3} \\
p_{x 4}
\end{array}\right]}_{p_{x}}=\underbrace{\left[\begin{array}{cccccc}
0 & -2-e & 1 & 0 & 0 & 0 \\
4+2 e & 0 & 0 & 0 & 0 & 0 \\
0 & 2 e & 2 & 0 & 0 & 0 \\
4-2 e & 0 & 0 & 0 & 0 & 0 \\
0 & 2-e & 1 & 0 & 0 & 0
\end{array}\right]}_{C_{x}} \vec{D},
$$




$$
\begin{aligned}
& \underbrace{\left[\begin{array}{c}
p_{y 0} \\
p_{y 1} \\
p_{y 2}
\end{array}\right]}_{p_{y}}=\underbrace{\left[\begin{array}{cccccc}
0 & 0 & 0 & 0 & 1 & 0 \\
0 & 0 & 0 & 0 & 0 & 2 \\
0 & 0 & 0 & 0 & -1 & 0
\end{array}\right]}_{C_{y}} \vec{D}, \\
& \underbrace{\left[\begin{array}{c}
p_{z 0} \\
p_{z 1} \\
p_{z 3} \\
p_{z 4}
\end{array}\right]}_{p_{z}}=\underbrace{\left[\begin{array}{cccccc}
e+1 & 0 & 0 & 2 & 0 & 0 \\
0 & 2 e+2 & 0 & 0 & 0 & 0 \\
-2 e & 0 & 0 & 4 & 0 & 0 \\
0 & 2-2 e & 0 & 0 & 0 & 0 \\
e-1 & 0 & 0 & 2 & 0 & 0
\end{array}\right]}_{C_{z}} \vec{D} .
\end{aligned}
$$

Before defining trajectory constraints, we first need to deal with the integral term $J(w)$ in the spacecraft relative motion equation (39). In order to have a rational expression for the motion, a polynomial approximation is derived to bound $J$ over $w \in\left[w_{k}, w_{k+1}\right]$ :

$$
J(w)=\Theta_{r}(w)+\varepsilon(w) \Rightarrow \underbrace{\Theta_{r}(w)-\bar{\varepsilon}}_{\Theta_{l}(w)} \leq J(w) \leq \underbrace{\Theta_{r}(w)+\bar{\varepsilon}}_{\Theta_{u}(w)}
$$

where $\Theta_{r}(w)$ is a polynomial of degree $r$ and $\bar{\varepsilon}$ the maximum error due to the approximation.

The linear constraints (34) are transformed by the change of variables (38) into:

$$
\begin{aligned}
A_{k} \overrightarrow{\tilde{\rho}}(w) \leq\left(\frac{1+e+(1-e) w^{2}}{1+w^{2}}\right) b_{k}, \forall w \in\left[w_{k} w_{k+1}\right], & \\
& \forall k=\{0, \ldots, N-1\},
\end{aligned}
$$

with $w_{k}=\tan \left(v_{k} / 2\right)$. To go further, let the $i^{\text {th }}$ row of this latter expression be expanded:

$$
A_{k_{i 1}} \tilde{x}(w)+A_{k_{i 2}} \tilde{y}(w)+A_{k_{i 3}} \tilde{z}(w) \leq\left(\frac{1+e+(1-e) w^{2}}{1+w^{2}}\right) b_{k_{i}},
$$

which is equivalent to:

$$
\frac{\Gamma_{i}^{k}(w)}{\left(1+w^{2}\right)^{2}} \geq 0
$$

with:

$$
\begin{aligned}
\Gamma_{i}^{k}(w)= & b_{k_{i}}\left(\left(1+w^{2}\right)\left(1+e+(1-e) w^{2}\right)\right)-A_{k_{i 1}}\left[P_{x}(w)+3 d_{4} P_{J x}(w) J(w)\right] \\
& -A_{k_{i 2}}\left(1+w^{2}\right) P_{y}(w)-A_{k_{i 3}}\left[P_{z}(w)+2 d_{4} P_{J z}(w) J(w)\right] .
\end{aligned}
$$


Replacing the function $J(w)$ by the two extreme bounding polynomials $\Theta_{l}$ and $\Theta_{u}$, the above function becomes polynomial, respectively $\Gamma_{i l}^{k}$ and $\Gamma_{i u}^{k}$. Hence, the inequality 47 becomes a pair of inequalities with $\Gamma_{i l}^{k}$ and $\Gamma_{i u}^{k}$, that must be repeated for each constraint $i$ (rows of $A_{k}$ ) and for each maneuver $k$. Finally, the whole constraint on the guidance error is formulated as the polynomial non negativity constraints:

$$
\left\{\begin{aligned}
0 \leq \Gamma_{i l}^{k}(w)= & b_{k_{i}}\left(\left(1+w^{2}\right)\left(1+e+(1-e) w^{2}\right)\right)-A_{k_{i 1}}\left[P_{x}(w)+3 d_{4} P_{J x}(w) \Theta_{l}(w)\right] \\
& -A_{k_{i 2}}\left(1+w^{2}\right) P_{y}(w)-A_{k_{i 3}}\left[P_{z}(w)+2 d_{4} P_{J z}(w) \Theta_{l}(w)\right], \\
0 \leq \Gamma_{i u}^{k}(w)= & b_{k_{i}}\left(\left(1+w^{2}\right)\left(1+e+(1-e) w^{2}\right)\right)-A_{k_{i 1}}\left[P_{x}(w)+3 d_{4} P_{J x}(w) \Theta_{u}(w)\right] \\
& -A_{k_{i 2}}\left(1+w^{2}\right) P_{y}(w)-A_{k_{i 3}}\left[P_{z}(w)+2 d_{4} P_{J z}(w) \Theta_{u}(w)\right],
\end{aligned}\right.
$$

for $i=\left\{1, \ldots, n_{c}\right\}$, for $k=\{0, \ldots, N-1\}, \forall w \in\left[w_{k} w_{k+1}\right]$.

\section{SDP formulation for the guidance error}

The properties of non negative polynomials and representation theorems of cones of non negative polynomials given in [14] are now used to translate these inequalities defined on an infinite interval into a semidefinite programming problem:

$$
\left\{\begin{array}{lll}
\exists Y_{1 i l}^{k}, Y_{2 i l}^{k} \geq 0 \quad \text { s.t. } & \gamma_{i l}^{k}=\Lambda^{*}\left(Y_{1 i l}^{k}, Y_{2 i l}^{k}\right), \\
\exists Y_{1 i u}^{k}, Y_{2 i u}^{k} \geq 0 \quad \text { s.t. } & \gamma_{i u}^{k}=\Lambda^{*}\left(Y_{1 i u}^{k}, Y_{2 i u}^{k}\right),
\end{array}\right.
$$

for $i=\left\{1, \ldots, n_{c}\right\}$, for $k=\{0, \ldots, N-1\} . \Gamma_{i l}^{k}$ and $\Gamma_{i u}^{k}$ are represented by their vector of coefficients $\gamma_{i l}^{k}$ and $\gamma_{i u}^{k}$, respectively. All $Y_{*}^{k}$ are square matrices whose dimensions depend on the order of the polynomials. The exact definition of the linear operator $\Lambda^{*}$ is omitted here for the sake of conciseness but it may be obtained in the appendix of the reference [12].

\section{Definition of the Cost function}

Apart from the control of the humps during the glideslope, the other main objective of the approach is to minimize the fuel consumption during the transfer. As 6 ungimbaled identical chemical thrusters are used, the cost function may be naturally defined as the 1-norm of the $N+1$ impulsive thrusts:

$$
\Upsilon(N)=\sum_{k=0}^{N}\left\|\Delta \vec{V}_{k}\right\|_{1}
$$


The formulation 51 is transformed in order to express the above criterion with respect to the decision variables $\vec{v}_{k_{+}}$, $k=0, \cdots, N-1, \lambda_{k}, k=1, \cdots, N-1$ and $\Delta \vec{V}_{N}$ :

$$
\Upsilon(N)=\left\|\vec{v}_{0_{+}}-\vec{v}_{0_{-}}\right\|_{1}+\sum_{k=1}^{N-1}\left\|\vec{v}_{k_{+}}-\Phi_{v \rho}^{[k-1]}\left(\vec{\rho}_{0}+\lambda_{k-1} \vec{u}_{g l s}\right)-\Phi_{v v}^{[k-1]} \vec{v}_{k-1_{+}}\right\|_{1}+\left\|\Delta \vec{V}_{N}\right\|_{1},
$$

where $\vec{v}_{0_{-}}$is the initial velocity vector. This cost function involving absolute values can be transformed into a linear function with the introduction of new variables and inequality constraints:

$$
\begin{array}{r}
\vec{v}_{0_{+}}-\vec{v}_{0_{-}} \leq \alpha_{0},-\left(\vec{v}_{0_{+}}-\vec{v}_{0_{-}}\right) \leq \alpha_{0} \\
\vec{v}_{k_{+}}-\Phi_{v \rho}^{[k-1]}\left(\vec{\rho}_{0}+\lambda_{k-1} \vec{u}_{g l s}\right)-\Phi_{v v}^{[k-1]} \vec{v}_{k-1_{+}} \leq \alpha_{k} \\
-\left(\vec{v}_{k_{+}}-\Phi_{v \rho}^{[k-1]}\left(\vec{\rho}_{0}+\lambda_{k-1} \vec{u}_{g l s}\right)-\Phi_{v v}^{[k-1]} \vec{v}_{k-1_{+}}\right) \leq \alpha_{k} \\
\Delta \vec{v}_{N} \leq \alpha_{N},-\Delta \vec{v}_{N} \leq \alpha_{N}
\end{array}
$$

where $\alpha_{k}$ are extra decision variables, and the cost function becomes $\Upsilon(N)=\sum_{k=0}^{N}\left[\begin{array}{lll}1 & 1 & 1\end{array}\right] \alpha_{k}$.

\section{E. A semidefinite programming problem}

After having defined all the different ingredients in the previous subsections, the last step consists in gathering them in a compact formulation. Therefore, a solution to the initial minimum-fuel glideslope guidance problem may be obtained via the solution of the semidefinite programming problem where the decision variables are : the auxiliary variables $\alpha_{k}$ for $k=\{0, \ldots, N\}$; the position parameters $\lambda_{k}$ for $k=\{1, \ldots, N-1\}$; the velocity vectors $\vec{v}_{k_{+}}$for $k=\{0, \ldots, N-1\}$; the last increment vector $\Delta \vec{V}_{N}$; the semidefinite matrices $Y_{1 i l}^{k}, Y_{2 i l}^{k}, Y_{1 i u}^{k}, Y_{2 i u}^{k}$ for $i=\left\{1, \ldots, n_{c}\right\}$, for $k=\{0, \ldots, N-1\}$. 


$$
\begin{aligned}
& \min c^{T} \alpha \\
& \text { s.t. } \\
& \lambda_{k+1} \vec{u}_{g l s}-\lambda_{k} \Phi_{\rho \rho}^{[k]} \vec{u}_{g l s}-\Phi_{\rho v}^{[k]} \vec{v}_{k_{+}}=\left(\Phi_{\rho \rho}^{[k]}-\mathcal{I}_{3}\right) \vec{\rho}_{0}, k=1, \ldots, N-1, \\
& \lambda_{1} \vec{u}_{g l s}-\Phi_{\rho v}^{[0]} \vec{v}_{0_{+}}=\left(\Phi_{\rho \rho}^{[0]}-\mathcal{I}_{3}\right) \vec{\rho}_{0}, \\
& \vec{v}_{f}-\Phi_{v \rho}^{[N-1]} \vec{\rho}_{0}=\Phi_{v \rho}^{[N-1]} \vec{u}_{g l s} \lambda_{N-1}+\Phi_{v v}^{[N-1]} \vec{v}_{N-1_{+}}+\Delta \vec{V}_{N}, \\
& \alpha_{0} \geq \vec{v}_{0_{+}}-\vec{v}_{0_{-}}, \\
& \alpha_{0} \geq-\left(\vec{v}_{0_{+}}-\vec{v}_{0_{-}}\right) \text {, } \\
& \alpha_{k} \geq \Phi_{v \rho}^{[k-1]}\left(\lambda_{k-1} \vec{u}_{g l s}+\rho_{0}\right)+\Phi_{v v}^{[k-1]^{2}} \vec{v}_{k-1_{+}}-\vec{v}_{k_{+}}, \\
& \alpha_{k} \geq \vec{v}_{k_{+}}-\Phi_{v \rho}^{[k-1]}\left(\lambda_{k-1} \vec{u}_{g l s}+\rho_{0}\right)-\Phi_{v v}^{[k-1]} \vec{v}_{k-1_{+}}, k=1, \ldots, N-1, \\
& \alpha_{N} \geq \Delta \vec{V}_{N} \\
& \alpha_{N} \geq-\Delta \vec{V}_{N}, \\
& \gamma_{i l}^{k}=\Lambda^{*}\left(Y_{1 i l}^{k}, Y_{2 i l}^{k}\right), i=\left\{1, \ldots, n_{c}\right\}, k=\{0, \ldots, N-1\}, \\
& \gamma_{i u}^{k}=\Lambda^{*}\left(Y_{1 i u}^{k}, Y_{2 i u}^{k}\right), \\
& Y_{1 i l}^{k} \geq 0, Y_{2 i l}^{k} \geq 0 \\
& Y_{1 i u}^{k} \geq 0, Y_{2 i u}^{k} \geq 0,
\end{aligned}
$$

with $c=\mathbb{1}_{3(N+1)}$ and $\alpha=\left[\begin{array}{ccc}\alpha_{0}^{T} & \ldots & \alpha_{N}^{T}\end{array}\right]^{T}$. When considering a second order polynomial approximation, we have that $Y_{1 i l}^{k} \in \mathbb{R}^{4 \times 4}, Y_{2 i l}^{k} \in \mathbb{R}^{3 \times 3}, Y_{1 i u}^{k} \in \mathbb{R}^{4 \times 4}$ and $Y_{2 i u}^{k} \in \mathbb{R}^{3 \times 3}$. Vectors $\gamma_{i l}^{k}$ and $\gamma_{i u}^{k}$ are the given vectors of coefficients of polynomials $\Gamma_{i l}^{k}$ and $\Gamma_{i u}^{k} . n_{c}$ is the number of rows of matrix $A_{k}$. This problem can hence be solved efficiently with an SDP solver. It is worthy to note that if constraints on the guidance error are removed, the optimization problem comes down to an LP problem.

\section{Simplified linear programming solutions for V-bar and R-bar approaches}

The goal here is to show that when the direction of the glideslope is defined to be the V-bar or the R-bar directions and for a circular reference orbit, the previous semidefinite programming problem may be simplified by using analytical developments to characterize the bounding corridor. Indeed, the main development is the same as for the previous section: we first define the glideslope line tracking constraints according to the classical approach. The maximum guidance error is then addressed by defining constraints on the humps profile and bounding them for both V-bar and R-bar cases. Finally, we propose a linear programming formulation leading to the obtention of a minimum-fuel solution to the glideslope guidance problem while controlling the guidance error for both V-bar and R-bar approaches. 


\section{A. V-bar and R-bar approaches}

In the literature, we find several missions that were performed following the V-bar classical approach, such as the ESA's ATV program [1] or the NASA's Space Shuttle mission [7]. Among the R-bar approach, Japan's HTV [5] or NASA's Cygnus program [3] may be distinguished.

These two common approach strategies are based on the directions of approaching a target in the close range phase of the rendezvous mission in a circular reference orbit, as shown in Figure 4 This is mainly due to observability LoS - constraints and safety reasons imposing the requirement of a trajectory belonging to a cone-shaped approach corridor.

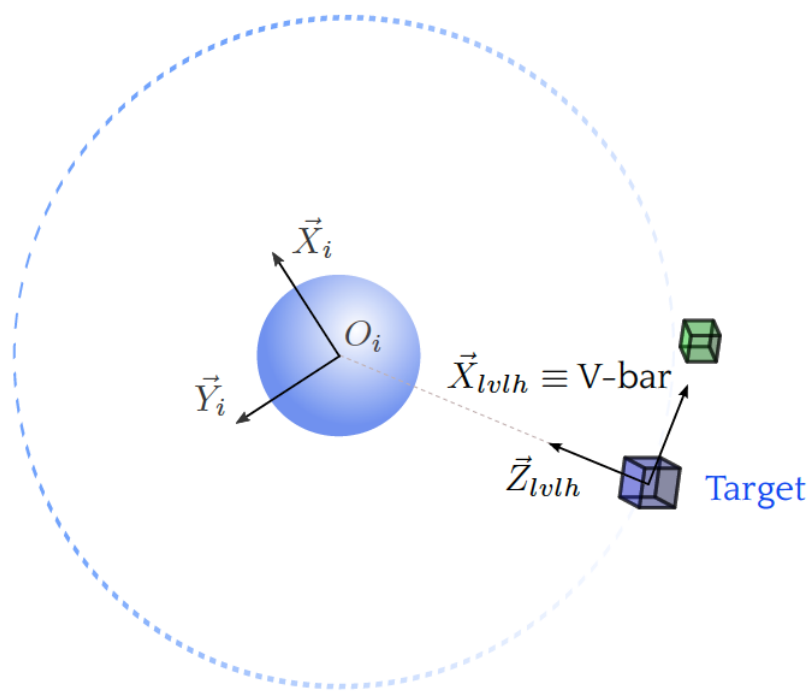

(a) V-bar approach.

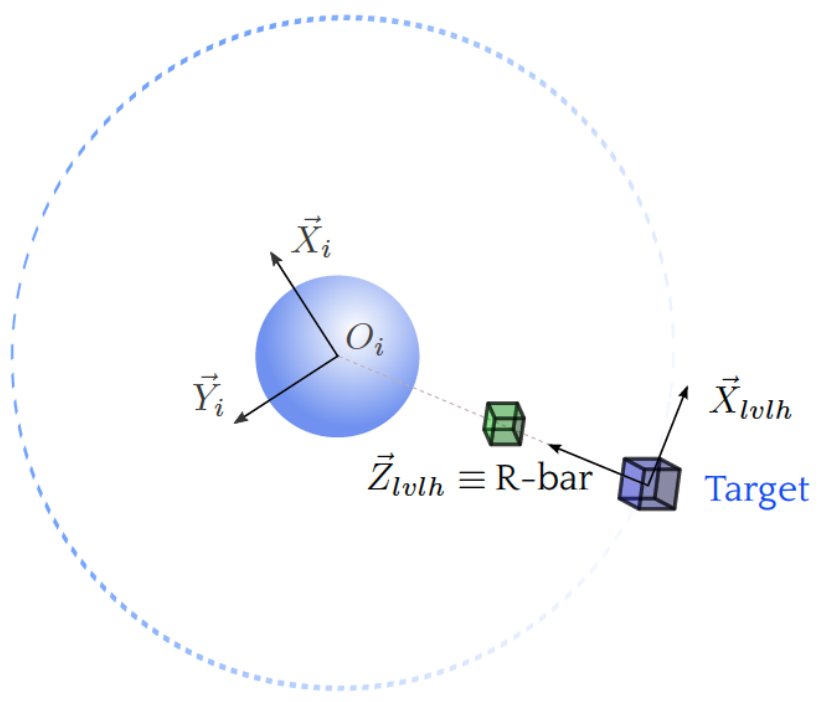

(b) R-bar approach.

Figure 4 Chaser satellite following a V-bar and R-bar glideslope approach.

V-bar and R-bar approaches are specific motions by which the chaser approaches the target in the tangential direction (V-bar) or radial direction (R-bar), meaning that the motion is restricted to the target's orbital plane. In the case of a circular orbit, the relative dynamics can be simplified by taking advantage of the fact that the dynamic matrix is Linear Time Invariant (LTI). By defining the relative position vector as $\vec{\rho}(t)=\left[\begin{array}{ll}x & z\end{array}\right]^{T}$ and the relative velocity as $\vec{v}(t)=\left[\begin{array}{ll}\dot{x} & \dot{z}\end{array}\right]^{T}$, the four partitions of $\Phi\left(t, t_{0}\right)$ have a simpler expression by just selecting the appropriate rows and columns. Having set the interval $\Delta t$, equal to $T / N$, the transition matrix is constant. We have therefore $\Phi_{\rho \rho}^{[k]}=\Phi_{\rho \rho}, \Phi_{\rho v}^{[k]}=\Phi_{\rho v}, \Phi_{v \rho}^{[k]}=\Phi_{v \rho}$ 
and $\Phi_{v v}^{[k]}=\Phi_{v v}$, as:

$$
\begin{array}{rlr}
\Phi_{\rho \rho}=\left[\begin{array}{cc}
1 & 6(n \Delta t-\sin (n \Delta t)) \\
0 & 4-3 \cos (n \Delta t)
\end{array}\right], & \Phi_{\rho v}=\left[\begin{array}{cc}
(4 / n) \sin (n \Delta t)-3 \Delta t & (2 / n)(1-\cos (n \Delta t)) \\
-(2 / n)(1-\cos (n \Delta t)) & \sin (n \Delta t) / n
\end{array}\right], \\
\Phi_{v \rho}=\left[\begin{array}{cc}
0 & 6 n(1-\cos (n \Delta t)) \\
0 & 3 n \sin (n \Delta t)
\end{array}\right], & \Phi_{v v}=\left[\begin{array}{cc}
-3+4 \cos (n \Delta t) & 2 \sin (n \Delta t) \\
-2 \sin (n \Delta t) & \cos (n \Delta t)
\end{array}\right],
\end{array}
$$

for all $k=0,1, \ldots, N-1$.

Depending on the choice for a V-bar approach or an R-bar approach, the relative position vector to be considered will be different. For a V-bar approach, the $z$ component along the $\vec{Z}_{o t}$ axis remains constant and equals its initial value, $z_{0}$, leading to:

$$
\vec{\rho}_{0}=\left[\begin{array}{c}
x_{0} \\
z_{0}
\end{array}\right], \quad \vec{\rho}_{k}=\left[\begin{array}{c}
x_{k} \\
z_{0}
\end{array}\right], \quad \vec{\rho}_{f}=\left[\begin{array}{c}
x_{f} \\
z_{0}
\end{array}\right] .
$$

On the other hand, for an R-bar approach, the $x$ component along the $\vec{X}_{o t}$ axis remains constant and equals $x_{0}$ :

$$
\vec{\rho}_{0}=\left[\begin{array}{c}
x_{0} \\
z_{0}
\end{array}\right], \quad \vec{\rho}_{k}=\left[\begin{array}{c}
x_{0} \\
z_{k}
\end{array}\right], \quad \vec{\rho}_{f}=\left[\begin{array}{c}
x_{0} \\
z_{f}
\end{array}\right] .
$$

Hereafter, considering a specific direction (V-bar or R-bar) and a circular reference orbit will significantly simplify the constraints on guidance error. On the other hand, the other features (glideslope line tracking, final velocity, cost function) remain basically of the same form.

\section{B. Constraints on guidance error}

In order to control the maximum guidance error, we first need to define the error in the orbital plane $\vec{\epsilon}$ in a general framework for both V-bar and R-bar approaches.

Let us define the point $A$ in the $x-z$ plane (see Figure 5) as the orthogonal projection of the chaser position $\vec{\rho}$ on the glideslope straight line. The excursion $\vec{\epsilon}$ is given by the difference between vectors $\vec{\rho}$ and $\vec{\rho}_{g l s}: \vec{\epsilon}(t)=\vec{\rho}(t)-\vec{\rho}_{g l s}(t)$. The auxiliary vector $\vec{\rho}_{g l s}$ can be itself expressed as:

$$
\vec{\rho}_{g l s}(t)=\vec{\rho}_{0}+\left(\Delta \vec{\rho}^{T} \vec{u}_{g l s}\right) \vec{u}_{g l s},
$$

where $\Delta \vec{\rho}=\vec{\rho}(t)-\vec{\rho}_{0}$ is also an auxiliary vector and $\vec{u}_{g l s}$ is the unit vector defining the glideslope straight line, defined 
in Equation (12), but for the in-plane case, it becomes:

$$
\vec{u}_{g l s}=\left[\begin{array}{lll}
\frac{x_{f}-x_{0}}{\lambda_{f}} & 0 & \frac{z_{f}-z_{0}}{\lambda_{f}}
\end{array}\right] .
$$

Hence, the excursion can be written as:

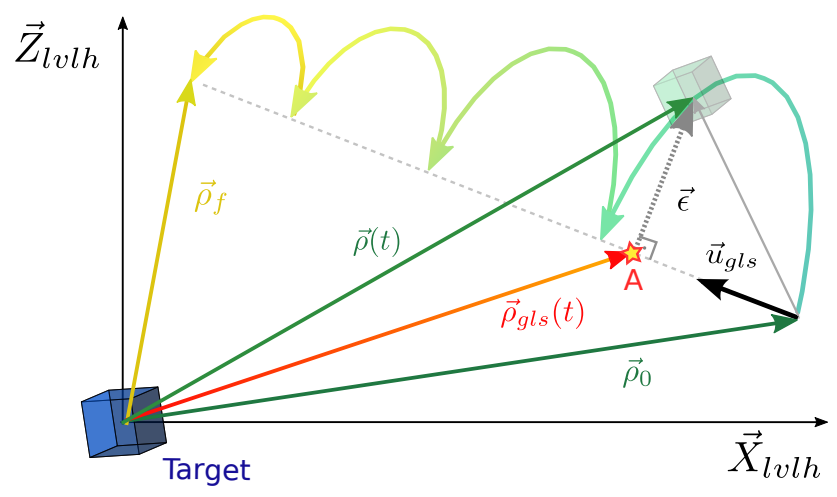

Figure 5 In-plane glideslope approach.

$$
\vec{\epsilon}(t)=\Delta \vec{\rho}-\left(\Delta \vec{\rho}^{T} \vec{u}_{g l s}\right) \vec{u}_{g l s},
$$

and the guidance error norm is then deduced as:

$$
\begin{aligned}
\|\vec{\epsilon}\|^{2} & =\left[\Delta \vec{\rho}-\left(\Delta \vec{\rho}^{T} \vec{u}_{g l s}\right) \vec{u}_{g l s}\right]^{T}\left[\Delta \vec{\rho}-\left(\Delta \vec{\rho}^{T} \vec{u}_{g l s}\right) \vec{u}_{g l s}\right], \\
& =\Delta \rho_{x}^{2}\left(1-u_{g l s_{x}}^{2}\right)+\Delta \rho_{z}^{2}\left(1-u_{g l s_{z}}^{2}\right)-2 u_{g l s_{x}} u_{g l s_{z}} \Delta \rho_{x} \Delta r_{z},
\end{aligned}
$$

with $\Delta \rho_{x}=x-x_{0}$ and $\Delta \rho_{z}=z-z_{0}$.

\section{Constraints on guidance error for V-bar}

If the approach is performed along a line parallel to the $x$-axis, the unit direction vector is:

$$
\vec{u}_{g l s}=\left[\begin{array}{lll}
1 & 0 & 0
\end{array}\right]^{T} .
$$

Hence, the guidance error norm in Expression 61) is reduced to:

$$
\|\vec{\epsilon}\|^{2}=\Delta \rho_{z}^{2} .
$$


Using the HCW $z$-equation, we get:

$$
\|\vec{\epsilon}\|^{2}=\left(3\left(1-\cos \left(n\left(t-t_{0}\right)\right)\right) z_{0}+\frac{2}{n}\left(\cos \left(n\left(t-t_{0}\right)\right)-1\right) \dot{x}_{0}+\frac{1}{n} \sin \left(n\left(t-t_{0}\right)\right) \dot{z}_{0}\right)^{2} .
$$

When the guidance error distance is maximal, the velocity vector $\vec{v}$ is parallel to the glideslope line. A maximum condition is then given by:

$$
\dot{z}=0 \Leftrightarrow 3 n \sin \left(n\left(t-t_{0}\right)\right) z_{0}-2 \sin \left(n\left(t-t_{0}\right)\right) \dot{x}_{0}+\cos \left(n\left(t-t_{0}\right)\right) \dot{z}_{0}=0 .
$$

Lemma 1 Let us define $\Delta t_{m}$ the value of the time at which the guidance error is maximal and $\Delta t$ the value of the time when guidance error is zero, that is, when the satellite is back on the glideslope commanded path. Considering a single hump, we have the following relationship:

$$
\Delta t=2 \Delta t_{m}
$$

meaning that the maximum occurs at the middle (in terms of time) of the hump. Proof. The proof is presented only in cases for which $n \Delta t \neq 2 k \pi, k=1,2, \ldots$. Consider Equation [65] on one hand and Equation $\|\vec{\epsilon}\|=0$ on the other hand. In both cases, $z_{0}$ can be expressed as:

$$
\begin{aligned}
& z_{0}=\frac{2}{3 n} \dot{x}_{0}-\frac{\cos \left(n \Delta t_{m}\right)}{3 n \sin \left(n \Delta t_{m}\right)} \dot{z}_{0}, \\
& z_{0}=\frac{2}{3 n} \dot{x}_{0}+\frac{\sin (n \Delta t)}{3 n(\cos (n \Delta t)-1)} \dot{z}_{0} .
\end{aligned}
$$

Equalizing the two equations leads to:

$$
\sin (n \Delta t) \sin \left(n \Delta t_{m}\right)=-\cos \left(n \Delta t_{m}\right) \cos (n \Delta t)+\cos \left(n \Delta t_{m}\right), \Leftrightarrow \cos \left(n \Delta t-n \Delta t_{m}\right)=\cos \left(n \Delta t_{m}\right) .
$$

Consequently, considering the time range only over a single hump, solutions of the above equation are $\Delta t=2 \Delta t_{m}$ and $\Delta t=0$.

From Equation (63), the guidance error distance can be readily upper-bounded as:

$$
\begin{aligned}
|\vec{\epsilon}| & =\left|(3-3 \cos (n \Delta t)) z_{0}+\frac{2}{n}(\cos (n \Delta t)-1) \dot{x}_{0}+\frac{1}{n} \sin (n \Delta t) \dot{z}_{0}\right| \\
& \leq\left|\left(3-3 \cos \left(n \Delta t_{m}\right)\right) z_{0}+\frac{2}{n}\left(\cos \left(n \Delta t_{m}\right)-1\right) \dot{x}_{0}+\frac{1}{n} \sin \left(n \Delta t_{m}\right) \dot{z}_{0}\right| .
\end{aligned}
$$

It provides an inequality that will be used to specify maximum conditions on guidance error for each hump, $k=$ $0,1, \ldots, N-1$ :

$$
\left|\left(3-3 \cos \left(n \Delta t_{m}\right)\right) z_{0}+\frac{2}{n}\left(\cos \left(n \Delta t_{m}\right)-1\right) \dot{x}_{k}+\frac{1}{n} \sin \left(n \Delta t_{m}\right) \dot{z}_{k}\right| \leq \delta_{k},
$$




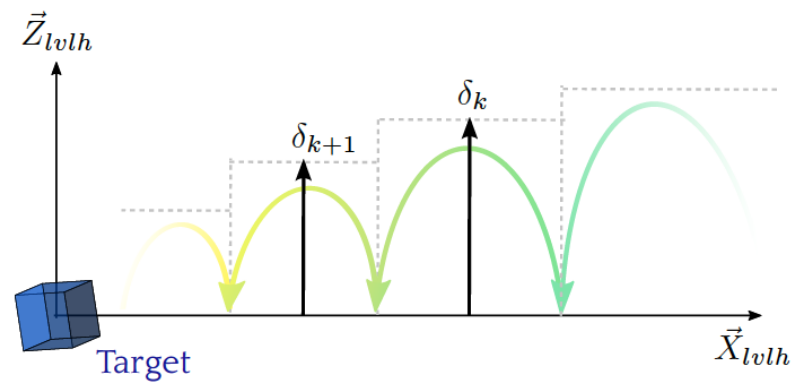

Figure 6 In-plane glideslope approach.

where $\delta_{k}$ is given and specifies the maximal allowable guidance error during the $(k+1)^{\text {th }}$ maneuver, as shown in Figure 6. Expression (70) is equivalently written as:

$$
\left\{\begin{array}{l}
\left(3-3 \cos \left(n \Delta t_{m}\right)\right) z_{0}+\frac{2}{n}\left(\cos \left(n \Delta t_{m}\right)-1\right) \dot{x}_{k}+\frac{1}{n} \sin \left(n \Delta t_{m}\right) \dot{z}_{k} \leq \delta_{k}, \\
-\left(3-3 \cos \left(n \Delta t_{m}\right)\right) z_{0}-\frac{2}{n}\left(\cos \left(n \Delta t_{m}\right)-1\right) \dot{x}_{k}-\frac{1}{n} \sin \left(n \Delta t_{m}\right) \dot{z}_{k} \leq \delta_{k} .
\end{array}\right.
$$

We recall that the interval $\Delta t$ being constant, the parameter $\Delta t_{m}$ is constant too. Being aware of the fact that $\vec{v}_{k_{+}}=\left[\begin{array}{ll}\dot{x}_{k} & \dot{z}_{k}\end{array}\right]^{T}$, the constraints (71) for the V-bar approach can be written in a matrix form as:

$$
M_{V} \vec{v}_{k_{+}} \leq M_{V_{k}}
$$

where:

$$
M_{V}=\left[\begin{array}{cc}
\frac{2}{n}\left(\cos \left(n \Delta t_{m}\right)-1\right) & \frac{1}{n} \sin \left(n \Delta t_{m}\right) \\
-\frac{2}{n}\left(\cos \left(n \Delta t_{m}\right)-1\right) & -\frac{1}{n} \sin \left(n \Delta t_{m}\right)
\end{array}\right], \quad M_{V_{k}}=\left[\begin{array}{c}
\delta_{k}-\left(3-3 \cos \left(n \Delta t_{m}\right)\right) z_{0} \\
\delta_{k}+\left(3-3 \cos \left(n \Delta t_{m}\right)\right) z_{0}
\end{array}\right]
$$

\section{Constraints on guidance error for R-bar}

If the approach is performed along a line parallel to the $z$-axis, the unit direction vector becomes:

$$
\vec{u}_{g l s}=\left[\begin{array}{lll}
0 & 0 & 1
\end{array}\right]^{T} .
$$

Hence, the guidance error norm in Expression 61) is reduced to:

$$
\|\vec{\epsilon}\|^{2}=\Delta \rho_{x}^{2}
$$


Using the HCW $x$-equation, we get:

$$
\begin{gathered}
\|\vec{\epsilon}\|^{2}=\left(6\left(n\left(t-t_{0}\right)-\sin \left(n\left(t-t_{0}\right)\right)\right) z_{0}+\frac{1}{n}\left(4 \sin \left(n\left(t-t_{0}\right)\right)-3 n\left(t-t_{0}\right)\right) \dot{x}_{0}\right. \\
\left.+\frac{2}{n}\left(1-\cos \left(n\left(t-t_{0}\right)\right)\right) \dot{z}_{0}\right)^{2} .
\end{gathered}
$$

Unlike the V-bar case, there is not a simple relationship between the time $\Delta t_{m}$ when the error is maximal and the maneuver interval $\Delta t$. Hence, a conservative bound is introduced.

Lemma 2 Let us define $\Delta t$ the value of the time when the guidance error is 0 , that is, when the satellite is back on the glideslope commanded path. Considering a single hump such that $n \Delta t \in\left[0 \arccos \frac{3}{4}\right]$, a conservative upper-bound of the guidance error is given by:

$$
|\vec{\epsilon}| \leq \tau_{1}\left|\dot{x}_{0}\right|+\tau_{2}\left|\dot{z}_{0}\right|+\tau_{3}\left|z_{0}\right|
$$

with: $\tau_{1}=\left|\frac{1}{n}(4 \sin (n \Delta t)-3 n \Delta t)\right| ; \tau_{2}=\left|\frac{2}{n}(1-\cos (n \Delta t))\right| ; \tau_{3}=\left|6\left(n \Delta t_{0}-\sin (n \Delta t)\right)\right|$.

Proof. Let us apply the triangle inequality to Expression (76:

$$
\begin{aligned}
|\vec{\epsilon}| \leq \mid 6\left(n\left(t-t_{0}\right)-\sin (\right. & \left.\left.n\left(t-t_{0}\right)\right)\right)|| z_{0}|+| \frac{1}{n}\left(4 \sin \left(n\left(t-t_{0}\right)\right)-3 n\left(t-t_{0}\right)\right)|| \dot{x}_{0} \mid \\
+ & \left|\frac{2}{n}\left(1-\cos \left(n\left(t-t_{0}\right)\right)\right)\right|\left|\dot{z}_{0}\right|
\end{aligned}
$$

Analyzing all terms separately, we have:

- First term, $\frac{d}{d\left(n\left(t-t_{0}\right)\right)}\left(n\left(t-t_{0}\right)-\sin \left(n\left(t-t_{0}\right)\right)\right)=1-\cos \left(n\left(t-t_{0}\right)\right) \geq 0$, hence the $1^{\text {st }}$ term $\leq \tau_{3}$.

- Second term, $\frac{d}{d\left(n\left(t-t_{0}\right)\right)}\left(4 \sin \left(n\left(t-t_{0}\right)\right)-3 n\left(t-t_{0}\right)\right)=4 \cos \left(n\left(t-t_{0}\right)\right)-3 \geq 0$, if $n\left(t-t_{0}\right) \leq \arccos \frac{3}{4}$, hence the $2^{\text {nd }}$ term $\leq \tau_{1}$.

- Third term, $\frac{d}{d\left(n\left(t-t_{0}\right)\right)}\left(1-\cos \left(n\left(t-t_{0}\right)\right)\right)=\sin \left(n\left(t-t_{0}\right)\right) \geq 0$, if $n\left(t-t_{0}\right) \leq \pi$, hence the $3^{\text {rd }}$ term $\leq \tau_{2}$.

$\square$ Although in the above lemma the range of $n \Delta t$ is restricted to [0 $\left.\arccos \frac{3}{4}\right]$, this result can be easily extended to a full revolution. The constraints [78] for the R-bar approach can be written in a matrix form as:

$$
M_{R} \vec{v}_{k_{+}} \leq M_{R_{k}}
$$

where:

$$
M_{R}=\left[\begin{array}{cc}
\tau_{1} & \tau_{2} \\
-\tau_{1} & -\tau_{2}
\end{array}\right], \quad M_{R_{k}}=\left[\begin{array}{c}
\delta_{k}-\tau_{3} z_{k} \\
\delta_{k}+\tau_{3} z_{k}
\end{array}\right]
$$

Note that the maximal allowable excursion at each step is denoted by $\delta_{k}$, which can be seen in Figure 6 with the corresponding permutation between the $X_{l v l h}$ and $Z_{l v l h}$ axes. 


\section{A linear programming problem}

The optimization problem is now formulated. The glideslope line tracking constraint 25$]$ and the final velocity constraint 29] require a slight adjustment. Positions on the glideslope $\vec{\rho}_{k}$ are now defined as 56 for V-bar, and 57 for R-bar (instead of 24). Blocks of the transition matrices $\Phi_{* *}^{[k]}$ reduce to $2 \times 2$ blocks 55 , constant w.r.t. $k$. The velocity decision variables are obviously restricted to the plane : $\vec{v}_{k_{+}}=\left[\begin{array}{ll}\dot{x}_{k} & \dot{z}_{k}\end{array}\right]^{T}$. Regarding the cost-related constraints $\left[53\right.$, the same adjustments hold, and variables $\alpha_{k} \in \mathbb{R}^{2}$ (instead of $\left.\mathbb{R}^{3}\right)$.

Gathering all the constraints with the appropriate decision variables, an LP problem is built for which a solution provides a minimum-fuel glideslope guidance along V-bar or R-bar:

$$
\begin{aligned}
& \min c^{T} \alpha \\
& \text { s.t. } \\
& \vec{\rho}_{k+1}=\Phi_{\rho \rho} \vec{\rho}_{k}+\Phi_{\rho v} \vec{v}_{k_{+}}, k=0, \ldots, N-1, \\
& \Delta \vec{V}_{N}=\vec{v}_{f}-\Phi_{v \rho} \vec{\rho}_{N-1}-\Phi_{v v} \vec{v}_{N-1_{+}}, \\
& \alpha_{0} \geq \vec{v}_{0_{+}}-\vec{v}_{0_{-}}, \alpha_{0} \geq-\left(\vec{v}_{0_{+}}-\vec{v}_{0_{-}}\right), \\
& \alpha_{k} \geq \Phi_{v \rho} \vec{\rho}_{k-1}+\Phi_{v v} \vec{v}_{k-1_{+}}-\vec{v}_{k_{+}}, \\
& \alpha_{k} \geq \vec{v}_{k_{+}}-\Phi_{v \rho} \vec{\rho}_{k-1}-\Phi_{v v} \vec{v}_{k-1_{+}}, k=1, \ldots, N-1, \\
& \alpha_{N} \geq \Delta \vec{V}_{N}, \alpha_{N} \geq-\Delta \vec{V}_{N}, \\
& M_{*} \vec{v}_{k_{+}} \leq M_{* k}, k=0, \ldots, N-1,
\end{aligned}
$$

with $c=\mathbb{1}_{2(N+1)}$ and $\alpha=\left[\begin{array}{lll}\alpha_{0}^{T} & \ldots & \alpha_{N}^{T}\end{array}\right]^{T}$. In the last inequality, $*=V$ or $*=R$ depending on the approach, and the corresponding matrices are defined in $\left(73\right.$ and $80 . \vec{\rho}_{k}$ is defined as 56) for V-bar, and 57) for R-bar. Let recall that scalars $\delta_{k}$ in $M_{*_{k}}$ are user-defined parameters that set the maximum allowable magnitude for each hump.

\section{Numerical examples}

\section{A. Example 1}

First, an illustration based on PRISMA [21] is presented. The PRISMA program is a cooperative effort between the Swedish National Space Board (SNSB), the French Centre National d'Etudes Spatiales (CNES), the German Deutsche Zentrum für Luft- und Raumfahrt (DLR) and the Danish Danmarks Tekniske Universitet (DTU) [2]. Launched on June 15, 2010 Yasny (Russia), it was intended to test in-orbit new guidance schemes (particularly autonomous orbit control) for formation flying and rendezvous technologies. The orbital elements of the target orbit, as well as initial and final rendezvous conditions, are listed in Table 1.

In this first example, the target evolves on a quasi-circular orbit and the standard glideslope approach is employed to 


\begin{tabular}{|c|c|c|}
\hline Semi-major axis & \multicolumn{2}{|r|}{$a=7011 \mathrm{~km}$} \\
\hline Inclination & \multicolumn{2}{|r|}{$i=98 \mathrm{deg}$} \\
\hline Argument of Perigee & \multicolumn{2}{|r|}{$\omega=0$ deg. } \\
\hline RAAN & \multicolumn{2}{|r|}{$\Omega=190 \mathrm{deg}}$. \\
\hline Eccentricity & \multicolumn{2}{|r|}{$e=0.004$} \\
\hline Initial True Anomaly & \multicolumn{2}{|r|}{$v_{0}=0 \mathrm{rad}$} \\
\hline$t_{0}$ & \multicolumn{2}{|r|}{$0 \mathrm{~s}$} \\
\hline$X_{0}^{T}=\left[\begin{array}{ll}\rho_{0}^{T} & v_{0_{-}}^{T}\end{array}\right]$ & {$\left[\begin{array}{ll}-400 & 40\end{array}\right.$} & $-50 \quad-0.5 \quad 0 \quad 0]] \mathrm{m}-\mathrm{m} / \mathrm{s}$ \\
\hline$T$ & \multicolumn{2}{|r|}{$1500 \mathrm{~s}$} \\
\hline$X_{f}^{T}=\left[\begin{array}{ll}\rho_{f}^{T} & v_{f}^{T}\end{array}\right]$ & {$\left[\begin{array}{ll}-40 & 0\end{array}\right.$} & $-10 \quad 0 \quad 0 \quad 0 \quad 0] \mathrm{m}-\mathrm{m} / \mathrm{s}$ \\
\hline$N$ & \multicolumn{2}{|r|}{6} \\
\hline
\end{tabular}

Table 1 PRISMA rendezvous characteristics.

transfer the chaser from $\vec{\rho}_{0}$ to $\vec{\rho}_{f}$ in 6 maneuvers $(N)$ during $25 \min (T=1500 \mathrm{~s})$. Three algorithms are compared: the classical one from [8] based on Hill-Clohessy-Wiltshire equations, its direct extension based on Yamanaka-Ankersen equations and the proposed optimal glideslope algorithm. In all cases, the resulting impulsive control sequence $\Delta \vec{V}_{i}$ is applied and propagated via the Tschauner-Hempel equations. We aim at emphasizing the significant drift induced when using the glideslope control computed with the Hill-Clohessy-Wiltshire equations even when the reference orbit is quasi-circular. Figure 7 shows the chaser trajectories for the different approaches. The final position of the chaser resulting from the Hablani's glideslope is $[-49.2,-0.2,-16] \mathrm{m}$, that is $11 \mathrm{~m}$ away from the planed final position. Regarding the velocity profile along the glideslope, the relevant parameters are set such that $\dot{\lambda}_{0}=-1$ and $\dot{\lambda}_{T}$ is imposed by the maneuver duration $T$. The global consumptions of the standard circular and elliptic glideslope algorithms are similar and given respectively by $2.9705 \mathrm{~m} / \mathrm{s}$ and $2.977 \mathrm{~m} / \mathrm{s}$. The specifications for the proposed algorithm are defined by a final velocity $\vec{v}_{f}=\overrightarrow{0} \mathrm{~m} / \mathrm{s}$ and a constraint on the trajectory characterized by a $10 \mathrm{~m} \times 10 \mathrm{~m}$ corridor. The obtained consumption is $1.9698 \mathrm{~m} / \mathrm{s}$ which is much lower than the standard approach.

The sequences of impulsive maneuvers for the standard elliptic glideslope and the minimum-fuel algorithm are detailed below in Table 2 and depicted in Figure 8 . Note also that the standard trajectories do not respect the admissible corridor in green.

\section{B. Example 2}

The PRISMA framework is now used to illustrate the results obtained for a V-bar approach in a circular orbit. Scenario parameters from Table 1 are identical, except for the eccentricity which is set to 0 to define a circular reference orbit. The out-of-plane component of the initial and final positions $\left(\rho_{0_{y}}\right.$ and $\left.\rho_{f_{y}}\right)$ are set to 0 , and the initial and final $z$-components must be equal: $\rho_{0_{z}}=\rho_{f_{z}}=-20 \mathrm{~m}$. The transfer time is set to $540 \mathrm{~s}$ for the sake of comparison with scenario 1 in $[8]$. The proposed optimal V-bar glideslope approach, the classical glideslope from $[8]$ and the 


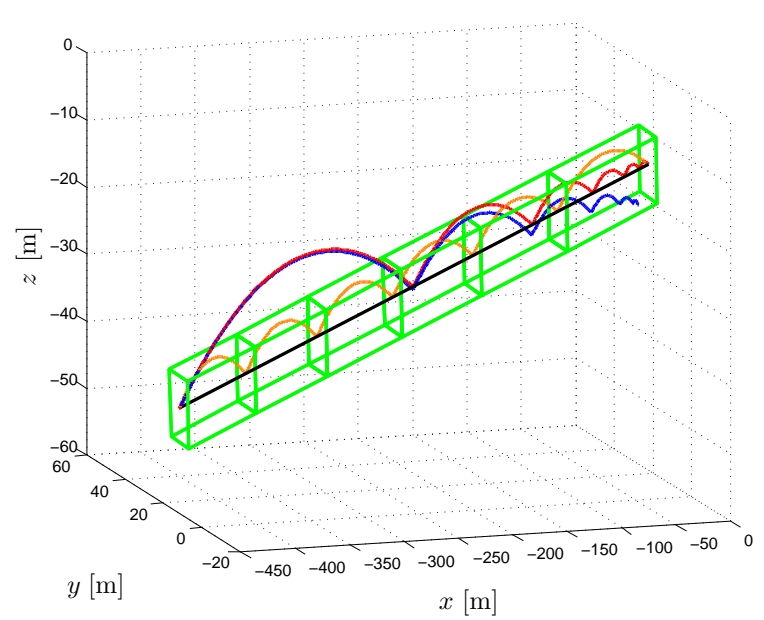

Figure 7 Chaser relative trajectories, standard circular glideslope (blue) vs. elliptic glideslope (red) vs. proposed optimal algorithm (orange).

\begin{tabular}{|l|ccccccc|}
\hline time (s) & 0 & 250 & 500 & 750 & 1000 & 1250 & 1500 \\
\hline \hline \multicolumn{2}{|l|}{ standard glideslope } \\
\hline$\Delta V_{x}(\mathrm{~m} / \mathrm{s})$ & 1.1784 & -0.3757 & -0.1915 & -0.0976 & -0.0498 & -0.0254 & -0.0245 \\
\hline$\Delta V_{y}(\mathrm{~m} / \mathrm{s})$ & -0.0749 & 0.0455 & 0.0231 & 0.0117 & 0.0058 & 0.0029 & 0.0028 \\
\hline$\Delta V_{z}(\mathrm{~m} / \mathrm{s})$ & 0.2879 & 0.2779 & 0.1455 & 0.0780 & 0.0436 & 0.0261 & 0.0083 \\
\hline \hline
\end{tabular}

Table 2 Impulsive control sequences for Example 1.

two-impulse method from [4] are compared on Figure 9] Regarding the proposed algorithm, three different sets of constraints on the maximum allowable excursion have been enforced (green lines). Results for each method are summarized in Table 3 The new algorithm reduces significantly the consumption while keeping the chaser close to the commanded path. As expected, the straightforward strategy from [4] is very efficient from the consumption point of view but is very weak regarding the guidance error.

In Figure 9a, the sequence of bounds on the trajectory is given by the vector $\left[\begin{array}{llllll}6 & 6 & 6 & 1 & 1 & 1\end{array}\right]$. While there is no constraint on the location of intermediate positions along the commanded path (order or distance, $x_{k}$ totally free), it is interesting to see that, for identical excursion constraints, the optimization naturally spaces them evenly. Besides, minimizing the consumption does not necessarily lead to a trade-off with guidance error. The first three humps do not reach the allowable limit. However, when the trajectory constraints become tighter on some maneuvers, humps

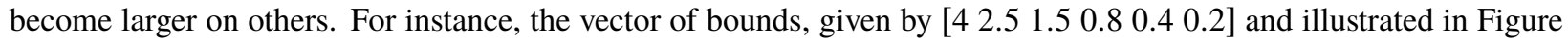



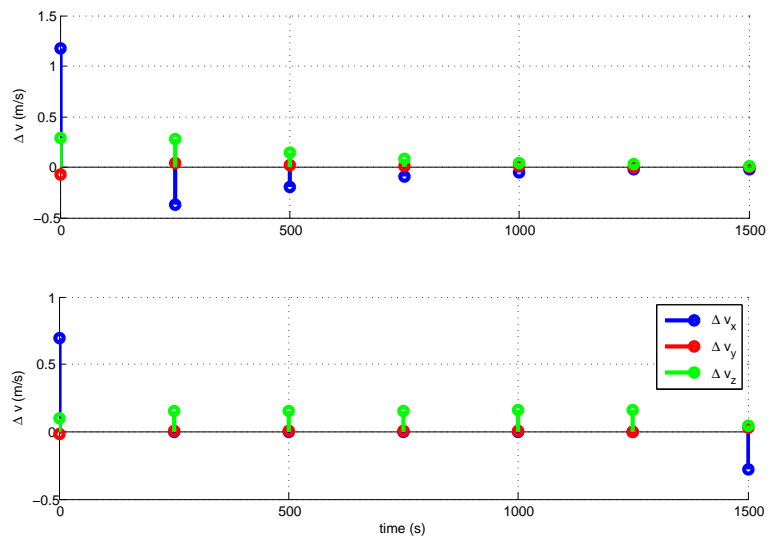

Figure 8 Impulsive control sequences for the standard elliptic glideslope (top) and for the optimal glideslope (bottom) in Example 1.

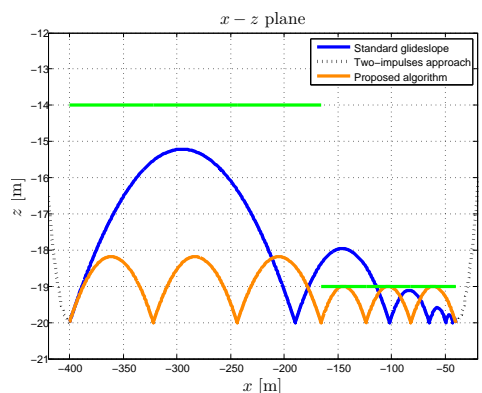

(a)

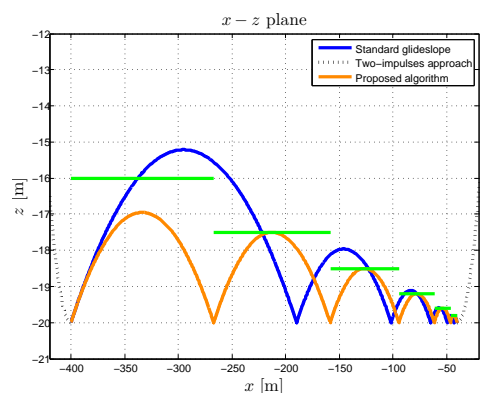

(b)

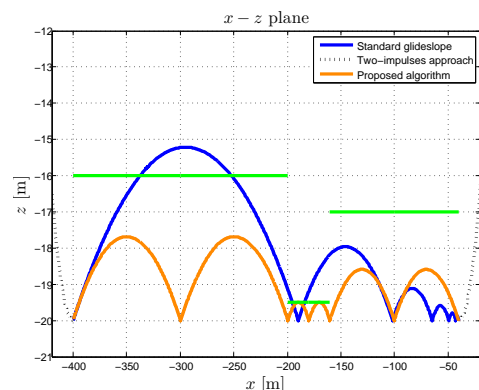

(c)

Figure 9 V-bar circular glideslope trajectories for different constraint sequences.

$9 b$ may typically arise from a LoS requirement. Demanding smaller guidance errors in the last maneuvers induces larger humps in the first ones. One can thus observe a waterbed effect where, for a given distance $\lambda_{f}$ to travel, short humps need to be offset by larger ones, the number of maneuvers $N$ being fixed. It thus appears that the optimal algorithm provides velocity increments such that the range of the excursion is related to the traveled distance along the glideslope (for each hump). Obviously, this is not true for the classical glideslope algorithm where large humps with close intermediate positions may occur. This observation can also be made in the third case in Figure $9 \mathrm{c}$ Indeed, the guidance error constraints make the "shaping" of the trajectory profile possible.

\section{Example 3}

In this third example, the standard elliptic glideslope approach as it may be built from the information obtained in [11] and the proposed optimal algorithm are compared in a case for which the eccentricity of the reference orbit is 


\begin{tabular}{|c||ccc|ccc|}
\hline \multicolumn{1}{|c||}{} & \multicolumn{3}{|c|}{ consumption $[\mathrm{m} / \mathrm{s}]$} & \multicolumn{3}{|c|}{ max. excursion $[\mathrm{m}]$} \\
\hline Simulations & (a) & (b) & (c) & (a) & (b) & (c) \\
\hline \hline Standard glideslope [8] & \multicolumn{3}{|c|}{5.87} & \multicolumn{3}{|c|}{4.78} \\
\hline Two-impulse method [4] & \multicolumn{3}{|c|}{0.5} & & \multicolumn{3}{|c|}{117} & \\
\hline optimal V-bar glideslope & 2.98 & 4.19 & 4.37 & 1.81 & 3.04 & 2.31 \\
\hline
\end{tabular}

Table 3 Simulation results for Example 2, (cf. Figure 9).

high. The parameters defining the conditions of the rendezvous are set as follows:

$$
\begin{aligned}
& T=540 \mathrm{~s}, N=4, n=0.001 \mathrm{rad} / \mathrm{s}, e=0.7, \vec{v}_{f}=\overrightarrow{0}, \\
& \vec{\rho}_{0}=\left[\begin{array}{c}
-500 \\
10 \\
30
\end{array}\right] \mathrm{m}, \vec{\rho}_{f}=\left[\begin{array}{c}
-100 \\
0 \\
20
\end{array}\right] \mathrm{m}, \vec{v}_{0_{-}}=\left[\begin{array}{c}
0 \\
0 \\
0.5
\end{array}\right] \mathrm{m} / \mathrm{s} .
\end{aligned}
$$

Regarding the constraints on the trajectory guidance error, we define for each maneuver a box (or a corridor) defined by 4 planes with (33). Thus, 4 boxes centered around the glideslope line are defined:

\begin{tabular}{lcccc} 
maneuver & 1 & 2 & 3 & 4 \\
\hline height [m] & 90 & 50 & 30 & 20 \\
width [m] & 10 & 10 & 10 & 10
\end{tabular}

Figure 10 depicts the chaser trajectories for the two methods and where the four boxes corresponding to trajectory constraints are represented in green. The consumption of the standard glideslope algorithm is $7.74 \mathrm{~m} / \mathrm{s}$ whereas the consumption of our optimal algorithm is $4.53 \mathrm{~m} / \mathrm{s}$. The respective sequences of impulsive maneuvers are presented in Table 4 and depicted in Figure 11

\section{Conclusions}

A revisited solution to the problem of the impulsive close range rendezvous along a glideslope line is proposed, first for any type of approach, and later on, in the specific cases of the V-bar and R-bar approaches. The motivations for the development of a new solution to the glideslope method come from two main issues regarding the standard algorithm: the uncontrolled humps, inherent to the impulsive method and the relative motion dynamics, and the fuel consumption that is assessed a posteriori. These shortcomings are addressed via a reformulation of the problem in an optimization framework. To this end, some degrees of freedom are introduced by setting free suitable variables. 


\begin{tabular}{|l|ccccc|}
\hline time $(\mathrm{s})$ & 0 & 135 & 270 & 405 & 540 \\
\hline \hline \multicolumn{2}{|l|}{ standard glideslope } \\
\hline$\Delta V_{x}(\mathrm{~m} / \mathrm{s})$ & 2.2830 & -1.5736 & -0.3350 & -0.0669 & -0.0165 \\
\hline$\Delta V_{y}(\mathrm{~m} / \mathrm{s})$ & -0.0512 & 0.0544 & 0.0095 & 0.0017 & 0.0004 \\
\hline$\Delta V_{z}(\mathrm{~m} / \mathrm{s})$ & 1.3608 & 1.6940 & 0.2323 & 0.0486 & 0.0114 \\
\hline \hline \multicolumn{6}{|l}{ optimal glideslope } \\
\hline$\Delta V_{x}(\mathrm{~m} / \mathrm{s})$ & 0.9751 & 0 & 0 & 0 & -0.6517 \\
\hline$\Delta V_{y}(\mathrm{~m} / \mathrm{s})$ & -0.0129 & 0.0168 & 0.0039 & 0.0009 & 0.0165 \\
\hline$\Delta V_{z}(\mathrm{~m} / \mathrm{s})$ & 0.5938 & 1.2648 & 0.5613 & 0.3079 & 0.1267 \\
\hline
\end{tabular}

Table 4 Impulsive control sequences for Example 3.

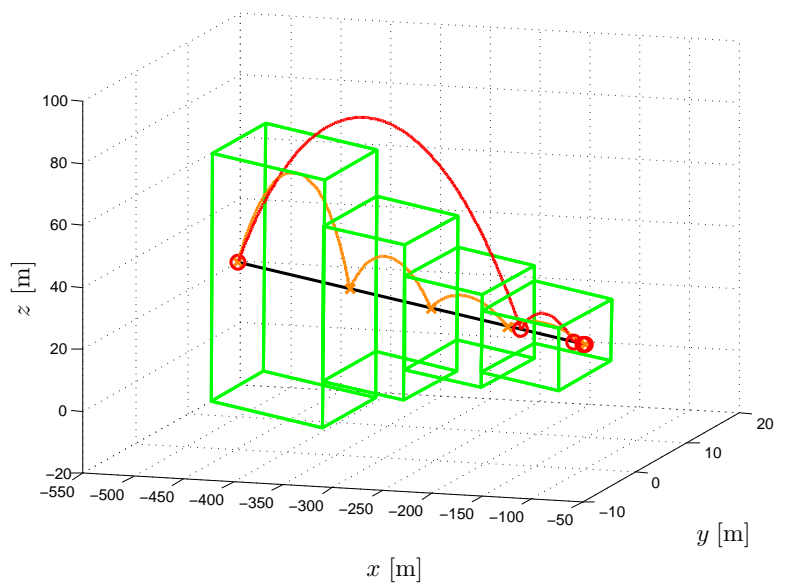

Figure 10 Chaser relative trajectories: standard elliptic glideslope algorithm (red) vs. proposed optimal algorithm (orange).

Then, combining an appropriate parametrization of the Tschauner-Hempel equations and a well-known result on non negative polynomials, the design of the glideslope guidance algorithm becomes a semidefinite programming problem.

The main design features included in the new proposed glideslope algorithm are on one hand the minimization of the consumption and, on the other hand, the possibility to specify an admissible volume for each hump of the relative trajectory and therefore to control the guidance error all along the rectilinear path. From this general case using an SDP formulation, and going further into the analysis with analytical expressions, we derived the solution via an LP problem for the specific cases of V-bar and R-bar approaches. This solution yields optimal consumption while respecting a user-defined maximum bound profile on the trajectory, which turns out to be very useful when dealing with visibility constraints while keeping a reasonable numerical computation complexity.

Different numerical examples illustrate the usefulness of the new methods compared to the classical one, especially when the approach corridor has to verify stringent geometrical restrictions such as LoS constraints. The consumption is also significantly reduced. 

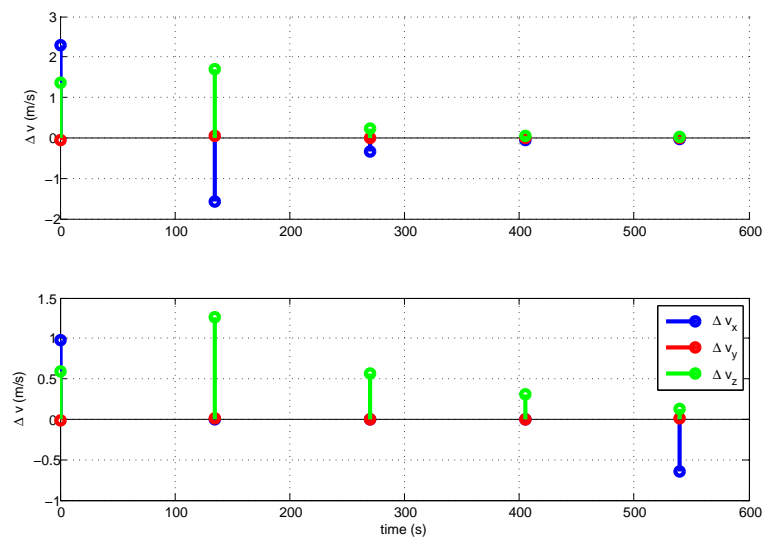

Figure 11 Impulsive control sequences for the standard glideslope (top) and for the optimal glideslope (bottom) in example 3.

Acknowledgments The authors would like to thank J.C. Berges from CNES Toulouse for the grants that partly supports this activity.

\section{References}

[1] Fabrega, J., Frezet, M., and Gonnaud, J.-L., “ATV GNC during rendezvous,” Proceedings of the Third International Conference on Spacecraft Guidance, Navigation and Control Systems, Noordwijk The Netherlands, 1996.

[2] Larsson, R., Berge, S., Bodin, P., and Jönsson, U., "Fuel efficient relative orbit control stategies for formation flying and rendezvous within Prisma," 29 $9^{\text {th }}$ Annual AAS Guidance and Control Conference, Breckenridge, Colorado, USA, 2006.

[3] Miotto, P., Breger, L., Mitchell, I., Keller, B., and Rishikof, B., "Designing and validating proximity operations rendezvous and approach trajectories for the Cygnus mission," AIAA Guidance, Navigation, and Control Conference, AIAA-2010-8446, Toronto, Ontario, Canada, 2009.

[4] Fehse, W. (ed.), Automated rendezvous and docking of spacecraft, Cambridge Aerospace Series, Cambridge University Press, Cambridge, UK, 2003.

[5] Kambara, N., Suzuki, M., Watabe, D., onji, M., Yoshikawa, S., Uematsu, H., Kasai, T., and Ueda, S., "Rendezvous technique of HTV and evaluation of on-orbit results," 62nd International Astronautical Congress, Cape Town, South Africa, 2011.

[6] Wen, C., and Gurfil, P., "Guidance, Navigation and Control for Autonomous R-bar Proximity Operations for Geostationary Satellites," Proceedings of Institution of Mechanical Engineers Part G: Journal of Aerospace Engineering, 2016.

[7] Pearson, D., “The glideslope approach,” Advances in Astronautical Sciences, Vol. 69, 1989, pp. 109-123.

[8] Hablani, H., Tapper, M., and David J. Dana-Bashian, D., "Guidance and relative navigation for autonomous rendezvous in a circular orbit," Journal of Guidance, Control and Dynamics, Vol. Vol. 25, No. No. 3, 2002. 
[9] Goodman, J., "Approaches and Fly-Arounds for Spacecraft Proximity Operations," Proceedings of the 27th Space Flight Mechanics Meeting, AAS 17-272, San Antonio, TX, USA, 2017.

[10] Wang, F., Cao, X., and Chen, X., "Guidance Algorithms for the Near-Distance Rendezvous of On-Orbit-Servicing Spacecraft," Transactions of Japanese Society for Aeronautical and Space Sciences, Vol. 50, No. 167, 2007, pp. 9-17.

[11] Okasha, M., and Newman, B., "Guidance, Navigation and Control for Satellite Proximity Operations using Tschauner-Hempel Equations," AIAA Guidance, Navigation and Control Conference, Portland, Oregon, USA, 2011.

[12] Deaconu, G., Louembet, C., and Théron, A., "Designing Continuously Constrained Spacecraft Relative Trajectories for Proximity Operations," Journal of Guidance, Control, and Dynamics, Vol. 38, No. 7, 2015, pp. 1208-1217.

[13] Yamanaka, K., and Ankersen, F., "New State Transition Matrix for Relative Motion on an Arbitrary Elliptical Orbit," Journal of Guidance, Control, and Dynamics, Vol. 25, 2002, pp. 60-66.

[14] Nesterov, Y., "Squared Functionals Systems and Optimization Problems," High Performance Optimization, edited by H. Frenck, K. Roos, T. Terlaky, and S. Zhang, Springer US, Boston, MA, USA, 2000, Chap. 17, pp. 405-440.

[15] Ariba, Y., Arzelier, D., Urbina Iglesias, L. S., and Louembet, C., "V-bar and R-bar Glideslope guidance algorithms for fixedtime rendre-vous: a linear programming approach," IFAC Symposium on Automatic Control in Aerospace (ACA), Sherbrooke (Canada), 2016.

[16] Ariba, Y., Arzelier, D., and Urbina Iglesias, L. S., "A New Glideslope Guidance Algorithm for Minimum-Fuel Fixed-Time Elliptic Rendezvous Using Semidefinite Programming," The 20th World Congress of IFAC, Toulouse (France), 2017.

[17] Alfriend, K., Vadali, S., Gurfil, P., How, J., and Breger, L., Spacecraft Formation Flying: Dynamics, Control and Navigation, Astrodynamics Series, Elsevier, Burlington, MA, USA, 2010.

[18] Lawden, D., Optimal trajectories for space navigation, Butterworth, London, England, 1963.

[19] Tschauner, J., “Elliptic orbit rendezvous,” AIAA Journal, Vol. 5, No. 6, 1967, pp. 1110-1113.

[20] Clohessy, W., and Wiltshire, R., “Terminal Guidance System for Satellite RendezVous,” Journal of the Astronautical Sciences, Vol. 27, No. 9, 1960, pp. 653-658.

[21] Berges, J.-C., Cayeux, P., Gaudel-Vacaresse, A., and Meyssignac, B., “CNES approaching guidance experiment on FFIORD,” 20th. International Symposium on Space Flight Dynamics, Annapolis, Maryland, USA, 2007. 